\title{
Degree Constrained Node-Connectivity Problems
}

\author{
Zeev Nutov
}

Received: date / Accepted: date

\begin{abstract}
We consider Degree Constrained Survivable Network problems. For the directed Degree Constrained $k$-Edge-Outconnected Subgraph problem, we slightly improve the best known approximation ratio, by a simple proof. Our main contribution is giving a framework to handle node-connectivity degree constrained problems with the iterative rounding method. In particular, for the degree constrained versions of the Element-Connectivity Survivable Network problem on undirected graphs, and of the $k$-Outconnected Subgraph problem on both directed and undirected graphs, our algorithm computes a solution $J$ of cost $O(\log k)$ times the optimal, with degrees $O\left(2^{k}\right) \cdot b(v)$. Similar result are obtained for the $k$-Connected Subgraph problem. The latter improves on the only degree approximation $O(k \log n) \cdot b(v)$ in $O\left(n^{k}\right)$ time on undirected graphs by Feder, Motwani, and Zhu.
\end{abstract}

Keywords Network design; Node-connectivity; Degree bounds; Approximation algorithms

\section{Introduction}

\subsection{Problem definition}

In Degree Constrained Connectivity Network Design problems, one seeks a cheap subgraph $J$ of a given graph $G$ that satisfies both connectivity requirements and degree constraints. Such problems are vastly studied in Combinatorial Optimization and Approximation Algorithms, see a recent survey by Lau, Ravi, and Singh [20]. One such type of problems are the matching/edge-cover problems, which are solvable in polynomial time. For other degree constrained problems, even checking whether there exists a feasible solution is NP-complete, hence one considers bicriteria approximation algorithms when the degree constraints are relaxed.

The Open University of Israel, E-mail: nutov@openu.ac.il 
We consider mainly degree constrained node-connectivity problems. In these problems we are given a directed/undirected graph $G=(V, E)$ with nonnegative edge-costs $\left\{c_{e}: e \in E\right\}$ and positive integral outdegree/degree bounds $b=\{b(v): v \in B \subseteq V\}$. The goal is to find a subgraph $J$ of $G$ that satisfies prescribed node-connectivity requirements, such that the degree/outdegree of every node $v$ is at most $b(v)$. In particular, we consider the following problems.

Degree Constrained $k$-Outconnected Subgraph

Here for a given integer $k$, the solution graph $J$ is required to be spanning and $k$-outconnected from a given root $s$, namely, $J$ should contain $k$ pairwise internally-disjoint paths from $s$ to every other node.

In the Degree Constrained $k$-Edge-Outconnected Subgraph problem, the paths should be only pairwise edge disjoint.

Degree Constrained $k$-Connected Subgraph

Here for a given integer $k$, the solution graph $J$ is required to be spanning and $k$-connected, namely, $J$ has at least $k+1$ nodes and should contain $k$ internally disjoint paths between every pair of its nodes.

In the above two problems, the input graph $G$ may be directed or undirected. In the case of directed graphs, one may consider also indegree constraints, where the indegree of every node $v$ should be at most $b^{\text {in }}(v)$.

In the next two problems, $G$ is assumed to be undirected, and we are given connectivity requirements $r=\{r(u, v): u, v \in U \subseteq V\}$ on a set $U$ of terminals.

Degree Constrained Element-Connectivity Survivable Network

Here for all $u, v \in U$, the solution graph $J$ should contain $r(u, v)$ pairwise edge disjoint $u v$-paths such that no two of them have a non-terminal node in $V \backslash U$ in common.

Degree Constrained Node-Connectivity Survivable Network

Here for all $u, v \in U$, the solution graph $J$ should contain $r(u, v)$ pairwise internally disjoint $u v$-paths. Rooted requirements is the case when there is $s \in U$ such that $r(u, v)>0$ implies $u=s$.

We say that an algorithm for a degree constrained connectivity problem is $(\alpha, \beta(b(v)))$-approximation, or that it has ratio $(\alpha, \beta(b(v)))$, if runs in polynomial time and outputs a solution such that its cost is at most $\alpha$ times the optimal value, and the degree (the out-degree, in the case of directed graph) of each $v \in B$ is at most $\beta(b(v))$, for any instance which has a feasible solution.

\subsection{Previous work and our results}

Degree constrained edge-connectivity problems were vastly studied. We refer the reader to a survey by Lau, Ravi, and Singh [20], and here only mention some literature relevant to this paper. One of the most important methods for 
approximating connectivity network design problems is the iterative rounding method, that was invented by Jain [14] to obtain ratio 2 for Edge-Connectivity Survivable Network. For degree-constrained edge-connectivity problems, this method was first applied by Singh and Lau [26] to obtain ratio $(1, b(v)+1)$ for the Degree Constrained Spanning Tree problem, and by Lau, Naor, Salavatipour, and Singh [18] to obtain ratio $(2,2 b(v)+3)$ for undirected Degree Constrained Edge-Connectivity Survivable Network; this was improved to $(2,2 b(v)+2)$ by Louis and Vishnoi [21], and to $(2, b(v)+6 k+3)$ by Lau and Singh [19], where $k$ denotes the maximum requirement.

For directed $k$-Edge-Outconnected Subgraph, Bansal, Khandekar, and Nagarajan [2] obtained a $\left(\frac{1}{\varepsilon},\left\lceil\frac{b(v)}{1-\varepsilon}\right\rceil+4\right)$-approximation scheme, $0 \leq \varepsilon \leq 1 / 2$. They also showed that this cost and degree approximation trade off cannot be much improved based on the standard LP-relaxation. For the version without costs, a $(b(v)+3)$-approximation is given in [23], and for $k=1 \mathrm{a}(b(v)+2)$ approximation is given in [2]. The version with indegree constraints admits an exact polynomial time algorithm [23]. Some additional results for related problems can be found in [2], [3], [11], and [23].

Our first result slightly improves by a simple and short proof the ratio $\left(\frac{1}{\varepsilon},\left[\frac{b(v)}{1-\varepsilon}\right]+4\right)$ for Degree Constrained $k$-Edge-Outconnected Subgraph of [2], matching for $\varepsilon=0$ the best known degree only approximation of [23].

Theorem 1 Directed Degree Constrained $k$-Edge-Outconnected Subgraph admits a $\left(\frac{1}{\varepsilon},\left\lceil\frac{b(v)}{1-\varepsilon}\right\rceil+3\right)$-approximation scheme, $\varepsilon \in[0.1 / 2)$.

However, there was only a small success in extending the iterative rounding method to node-connectivity degree constrained problems. Some previous work is as follows. Feder, Motwani, and Zhu [6] considered the undirected Degree Constrained $k$-Connected Subgraph problem (without costs), and gave an $O\left(n^{k}\right)$ time algorithm that computes a $k$-connected spanning subgraph $J$ of $G$ with degrees $O(k \log n) \cdot b(v)$; their algorithm does not rely on iterative rounding. In [15] is given a $(4,5 b(v)+3)$-approximation algorithm for $k=2$. the input graph is complete and the costs are metric is considered in [3].

Without degree constraints, Directed $k$-Outconnected Subgraph admits a polynomial time algorithm by Frank and Tardos [10], while other problems we consider are NP-hard. The currently best known cost approximation ratios for these problems are as follows: 2 for undirected $k$-Outconnected Subgraph [10] and Edge/Element-Connectivity Survivable Network $[14,7,4] ; O\left(\log k \cdot \log \frac{n}{n-k}\right)$ for $k$-Connected Subgraph for both directed and undirected graphs [22]; for Node-Connectivity Survivable Network on undirected graphs the best (nontrivial) ratios are $O\left(k^{3} \log |U|\right)$ for arbitrary requirements [5], and $O(k \log k)$ for rooted requirements [24].

Using an idea from [15] where the case $k=2$ is considered, we give a framework to handle node-connectivity degree constrained problems with the iterative rounding method, and obtain the following results. 
Theorem 2 For both directed and undirected graphs, Degree Constrained $k$ Outconnected Subgraph admits an $\left(O(\log k), O\left(2^{k}\right)\right)$-approximation algorithm. For directed graphs and indegree constraints, the problem admits an exact polynomial time algorithm.

Theorem 3 Degree Constrained Element-Connectivity Survivable Network admits a polynomial time algorithm that returns a subgraph $J$ of $G$ that satisfies the connectivity requirements, of cost $c(J)=O(\log k) \cdot \tau$ and degrees $O(k+\log k \cdot b(v))$ for all $v \in U$, and $O\left(2^{k}\right) \cdot b(v)$ for all $v \in V \backslash U$.

Recently, Fukunaga and Ravi [12] obtained ratios $(2,2 b(v)+4 k-1)$ for Degree Constrained $k$-Outconnected Subgraph and $(4 k-1,(4 k-1) b(v)+4 k-2)$ for Degree Constrained Element-Connectivity.

Our other results are deduced from Theorems 1, 2, and 3, using essentially known reductions. Using Theorem 2 we prove the following.

Theorem 4 If Degree Constrained $k$-Outconnected Subgraph admits approximation ratio $(\gamma, \rho(b(v)))$ then Degree Constrained $k$-Connected Subgraph admits approximation ratio $\left(\gamma+p k-1, \rho(b(v))+2 k^{2}\right)$, where $p=1$ for undirected graphs and $p=2$ for directed graphs. Thus for both directed and undirected graphs, Degree Constrained $k$-Connected Subgraph admits ratio $\left(O(k), O\left(2^{k}\right)\right)$.

This improves the $(\infty, O(\log n) \cdot b(v))$-approximation in $O\left(n^{k}\right)$ time for undirected graphs by [6], that does not rely on iterative rounding; for constant $k$ our degree approximation is a constant while that of [6] is $O(\log n)$, and for $k=O(\log \log n)$ our approximation is $O(\log n)$, while the algorithm of [6] has in this case running time $\Omega\left(n^{\log \log n}\right)$, which is not polynomial.

Chuzhoy and Khanna [5] showed that an instance of a node-connectivity problem can be decomposed into $p$-instances of element-connectivity problems, where $p=O\left(k^{3} \log |U|\right)$; in the case of rooted requirements $p=O\left(k^{2} \log |U|\right)$. This decomposition also applies for degree constrained problems. Combined with Theorem 3, we obtain the following.

Corollary 1 Degree Constrained Node-Connectivity Survivable Network admits a polynomial time algorithm that returns a subgraph $J$ of $G$ that satisfies the connectivity requirements, of cost $c(J)=p(k, n) \cdot O(\log k) \cdot \tau$ and with degrees: $p(k, n) \cdot O(k+\log k \cdot b(v))$ for all $v \in U$ and $p(k, n) \cdot O\left(2^{k}\right) \cdot b(v)$ for all $v \in V \backslash U$, where $p(k, n)=O\left(k^{3} \log |U|\right)$ in the case of arbitrary requirements and $p(k, n)=O\left(k^{2} \log |U|\right)$ in the case of rooted requirements.

The ratios in Theorems 2, 3, and 4 are rough, and for small values of $k$ we can obtain improved ratios, as follows.

Theorem 5 (i) Directed Degree Constrained 2-Outconnected Subgraph admits a $\left(2+\frac{1}{2 \varepsilon}, 2 b(v)+2\left\lceil\frac{b(v)}{2(1-\varepsilon)}\right\rceil+7\right)$-approximation scheme, $\varepsilon \in[0,1 / 2)$.

(ii) Directed Degree Constrained 2-Connected Subgraph admits a $\left(4+\frac{1}{2 \varepsilon}, 2 b(v)+2\left\lceil\frac{b(v)}{2(1-\varepsilon)}\right\rceil+9\right)$-approximation scheme, $\varepsilon \in[0,1 / 2)$. 
(iii) On undirected graphs, Degree Constrained 2-Outconnected Subgraph and Degree Constrained 2-Connected Subgraph admit ratio $(5,3 b(v)+9)$.

(iii) For $k=2$, Degree Constrained Element-Connectivity and Degree Constrained Node-Connectivity admit ratio $(4,4 b(v)+8)$.

This paper is organized as follows. In Sections 2, 3, 5 we prepare some tools that we use. We first formulate Theorems 1,2,3 in terms of a biset/setpair LPrelaxation (Section 2, Theorems 6,7,8), and then in terms of extreme point solutions of appropriate polytopes (Section 3, Theorems 9,10,11); in Sections 3 and 5 we establish some general properties of these extreme point solutions. Theorems 9,10,11 are then formally proved in Sections 4,6,7. In Section 8 we finish the proofs of Theorems 2,3,4, while Theorem 5 is proved in Section 9.

\section{Biset function edge-cover formulation of connectivity problems}

An edge e covers a set $S \subset V$ if it goes from $V \backslash S$ to $S$. For edge-connectivity problems, connectivity requirements can be specified by a set function $f$ on $V$, meaning that for every $S \subseteq V$ at least $f(S)$ edges should cover $S$. For example, by Menger's Theorem, a directed/undirected graph $J$ is $k$-edge-outconnected from $s$ if and only if at least $k$ edges of $J$ cover $S$ (namely, $f(S)=k$ ) for any non-empty subset of $V \backslash\{s\}$. A similar formulation for node-connectivity problems (c.f. $[9,7,4,8])$ relies on a known concept of "setpair" or "biset", as given in the following definition.

Definition 1 A biset is an ordered pair $\hat{S}=\left(S, S^{+}\right)$of subsets of a groundset $V$ such that $S \subseteq S^{+} ; S$ is the inner part and $S^{+}$is the outer part of $\hat{S}$, and $\Gamma(\hat{S})=S^{+} \backslash S$ is the boundary of $\hat{S}$. Let $\mathcal{V}$ denote the family of all bisets on $V$. The intersection and the union of bisets $\hat{X}, \hat{Y}$ is defined by $\hat{X} \cap \hat{Y}=$ $\left(X \cap Y, X^{+} \cap Y^{+}\right)$and $\hat{X} \cup \hat{Y}=\left(X \cup Y, X^{+} \cup Y^{+}\right)$. The biset $\hat{X} \backslash \hat{Y}$ is defined by $\hat{X} \backslash \hat{Y}=\left(X \backslash Y^{+}, X^{+} \backslash Y\right)$.

Any set $S$ can be considered as a biset $(S, S)$. To any biset $\left(S, S^{+}\right)$corresponds the setpair $\left(S, V \backslash S^{+}\right)$, c.f. [7,4]. An edge $e$ covers a biset $\hat{S}=\left(S, S^{+}\right)$if it goes from $V \backslash S^{+}$to $S$. For example, by Menger's Theorem, a directed graph $J$ is $k$-outconnected from $s$ if and only if at least $k-|\Gamma(\hat{S})|$ edges of $J$ cover $S$ (namely, $f(S)=k-|\Gamma(\hat{S})|$ ) for any biset $\hat{S}$ with $S \neq \emptyset$ and $S^{+} \subseteq V \backslash\{s\}$.

Definition 2 A biset function is (positively) intersecting supermodular if for any two bisets $\hat{X}, \hat{Y} \in \mathcal{V}$ with $f(\hat{X}), f(\hat{Y})>0$ which inner parts intersect, $f$ satisfies the supermodular inequality

$$
f(\hat{X})+f(\hat{Y}) \leq f(\hat{X} \cap \hat{Y})+f(\hat{X} \cup \hat{Y}) .
$$

A biset function $f$ on $V$ is (positively) weakly supermodular if for any two bisets $\hat{X}, \hat{Y} \in \mathcal{V}$ with $f(\hat{X}), f(\hat{Y})>0$ the function $f$ satisfies the inequality

$$
f(\hat{X})+f(\hat{Y}) \leq \max \{f(\hat{X} \cap \hat{Y})+f(\hat{X} \cup \hat{Y}), f(\hat{X} \backslash \hat{Y})+f(\hat{Y} \backslash \hat{X})\} .
$$


For a directed/undirected edge-set or a graph $J$ and $\hat{S} \in \mathcal{V}$ let $\delta_{J}^{\text {in }}(\hat{S})$ denote the set of edges in $J$ covering the biset $\hat{S}$; this notation is used for both directed and undirected graphs. For a node $v \in V$ let $\delta_{J}(v)$ denote the set of edges in $J$ leaving $v$ and $\operatorname{deg}_{J}(v)=\left|\delta_{J}(v)\right|$ the outdegree/degree of $v$ in $J$. Given a biset function $f$ on $V$, we say that a graph $J$ on $V$ is $f$-connected if $\left|\delta_{J}^{i n}(\hat{S})\right| \geq f(\hat{S})$ for all $\hat{S} \in \mathcal{V}$.

Let $\mathbb{R}_{+}$and $\mathbb{Z}_{+}$denote the set of positive reals and positive integers, respectively. We consider the following problem for both directed and undirected graphs with intersecting supermodular biset function $f$, and for undirected graphs with weakly supermodular $f$.

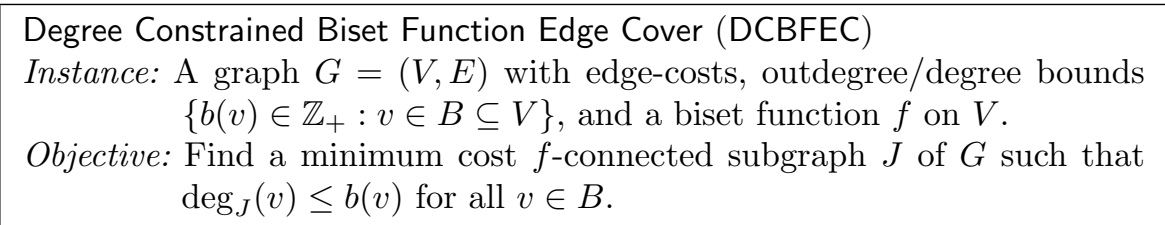

We assume that $G$ is $f$-connected, so $f(\hat{S})>0$ implies $S \neq \emptyset$ and $S^{+} \neq V$. In the case of the directed $k$-Outconnected Subgraph problem, $f$ is defined by $f(\hat{S})=\max \{k-|\Gamma(\hat{S})|, 0\}$ for bisets on $V \backslash\{s\}$ and $f(\hat{S})=0$ otherwise. This biset function $f$ is intersecting supermodular, see [8].

In the case of undirected Element Connectivity problem, $f$ is defined by $f(\hat{S})=\max \{r(\hat{S})-|\Gamma(\hat{S})|, 0\}$, where $r(\hat{S})$ is the requirement of $\hat{S}$ defined by $r(\hat{S})=\max \left\{r(u, v):|\{u, v\} \cap S|=\left|\{u, v\} \backslash S^{+}\right|=1\right\}$ if $U \cap S \neq \emptyset$, $U \backslash S^{+} \neq \emptyset$, and $\Gamma(\hat{S}) \subseteq U$; otherwise, $r(\hat{S})=0$. This biset function $f$ is weakly supermodular, see $[7,4]$.

Given a set $F$ and $x \in \mathbb{R}^{F}$ let $x(F)=\sum_{e \in F} x_{e}$. In DCBFEC problems, the function $f$ may not be given explicitly, but we assume that one can compute in polynomial time an extreme point optimal solution to the natural LP-relaxation $\tau=\min \{c \cdot x: x \in P(f, b, E)\}$ for DCBFEC, where $P(f, b, E)$ is the polytope defined by the following constraints:

\begin{tabular}{|c|c|}
\hline$x\left(\delta_{E}^{i n}(\hat{S})\right) \geq f(\hat{S})$ & for all $\hat{S} \in \mathcal{V}$ \\
\hline$x\left(\delta_{E}(v)\right) \leq b(v)$ & for all $v \in B$ \\
\hline $0 \leq x_{e} \leq 1$ & for all $e \in E$ \\
\hline
\end{tabular}

This assumption holds for all the problems considered; we omit the somewhat standard implementation details.

In the case of directed graphs and indegree constraints we consider the polytope $P\left(f, b^{i n}, E\right)$ with indegree constraints $x\left(\delta_{E}^{i n}(v)\right) \leq b^{i n}(v)$ instead of the outdegree constraints $x\left(\delta_{E}(v)\right) \leq b(v)$.

Since the $k$-Edge-Outconnected Subgraph problem is modeled by an intersecting supermodular set function, the following theorem implies Theorem 1.

Theorem 6 (Implies Theorem 1) Directed Degree Constrained Set Function Edge-Cover with intersecting supermodular set function $f$ admits $a\left(\frac{1}{\varepsilon},\left\lceil\frac{b(v)}{1-\varepsilon}\right\rceil+3\right)$ approximation scheme, $\varepsilon \in[0,1 / 2)$. 
Our degree approximation ratios for DCBFEC problems are expressed in terms of the following parameters. The role that these parameters play in our algorithms is briefly explained below, and for more details see Section 8 .

Definition 3 We say that bisets $\hat{X}, \hat{Y}$ are disjoint if $X \cap Y=\emptyset ; \hat{X}, \hat{Y}$ are strongly disjoint if $X \cap Y^{+}, Y \cap X^{+}=\emptyset$. For a biset function $f$ on $V$ let $\Delta_{f}(v)$ $\left(\Delta_{f}^{*}(v)\right)$ be the maximum size of a biset family $\mathcal{F} \subseteq\{\hat{S} \in \mathcal{V}: f(\hat{S})>0\}$ of pairwise disjoint (pairwise strongly disjoint) bisets such that $v \in \Gamma(\hat{S})$ for every $\hat{S} \in \mathcal{F}$. A biset function $f$ on $V$ is symmetric if $f(\hat{S})=f\left(\left(V \backslash S^{+}, V \backslash S\right)\right)$ for every $\hat{S} \in \mathcal{V}$.

In Section 8 we show that the following two theorems imply Theorems 2,3.

Theorem 7 (Implies Theorem 2) DCBFEC with intersecting supermodular biset function $f$ admits ratios $\left(2,2 b(v)+\Delta_{f}(v)+1\right)$ for directed graphs and $\left(4,2 b(v)+\Delta_{f}(v)+1+k\right)$ for undirected graphs, where $k=\max _{\hat{S} \in \mathcal{V}} f(\hat{S})$. In the case of directed graphs and indegree constraints, the problem admits an exact polynomial time algorithm.

Theorem 8 (Implies Theorem 3) Degree Constrained Biset Function EdgeCover admits approximation ratio $\left(3,3 b(v)+\max \left\{\Delta_{f}^{*}(v), 3\right\}+1\right)$ for undirected graphs and weakly supermodular symmetric biset-fumction $f$.

The symmetry assumption in Theorem 8 is not a restrictive one, and it is needed only for a correct evaluation of the parameter $\Delta_{f}^{*}(v)$; for a nonsymmetric $f$, our proof gives a degree approximation larger by one.

If $f$ is a set function, then $\Delta_{f}(v)=\Delta_{f}^{*}(v)=0$, and then better degree approximations are known than the ones in Theorems 7,8 . However in the case of biset functions, we seek to minimize the factor multiplying the terms $\Delta_{f}(v), \Delta_{f}^{*}(v)$ in the degree approximation, since these terms can be much larger than $b(v)$; even when all bisets $\hat{S}$ with positive $f$-value have $|\Gamma(\hat{S})|=1$, we may have $\Delta_{f}(v)=\Delta_{f}^{*}(v)=|V|-1$; an example is the biset function defined by $f(\hat{S})=1$ if $\hat{S} \in\{(u,\{u, v\}): u \in V \backslash\{v\}\}$ and $f(\hat{S})=0$ otherwise.

We now briefly explain how we use Theorem 7 to prove the degree approximation in Theorem 2; Theorem 3 is deduced from Theorem 8 in a similar way (for details see Section 8). Consider the version of the Degree Constrained $k$-Outconnected Subgraph problem when we seek to augment a graph $J$ which is $(\ell-1)$-outconnected from $s$ by a minimum cost edge set $I$ such that $J \cup I$ is $\ell$-outconnected from $s$, and such that $\operatorname{deg}_{I}(v) \leq b(v)$ for all $v \in V$. This problem can be formulated as DCBFEC with intersecting supermodular 0,1valued biset function $f_{\ell}$. Now consider the following sequential algorithm: start with $J=\emptyset$, and at iteration $\ell=1, \ldots, k$ add to $J$ an augmenting edgeset $I_{\ell}$ that covers $f_{\ell}$, using the algorithm for DCBFEC as in Theorem 2. We prove that $\Delta_{f_{\ell}}(v) \leq \operatorname{deg}_{J}(v)$ holds at the beginning of every iteration, hence $\operatorname{deg}_{I_{\ell}}(v) \leq 2 b(v)+\Delta_{f_{\ell}}(v)+1 \leq 2 b(v)+\operatorname{deg}_{J}(v)+1$. Denoting $d_{0}(v)=0$, we get the following recursive formula for the degree $d_{\ell}(v)$ of a node $v$ at the end of iteration $\ell: d_{\ell}(v)=d_{\ell-1}(v)+\operatorname{deg}_{I_{\ell}}(v) \leq 2 b(v)+2 d_{\ell-1}(v)+1$. Unraveling, we get the bound $d_{k}(v)=O\left(2^{k}\right) \cdot b(v)$ of Theorem 2 . 


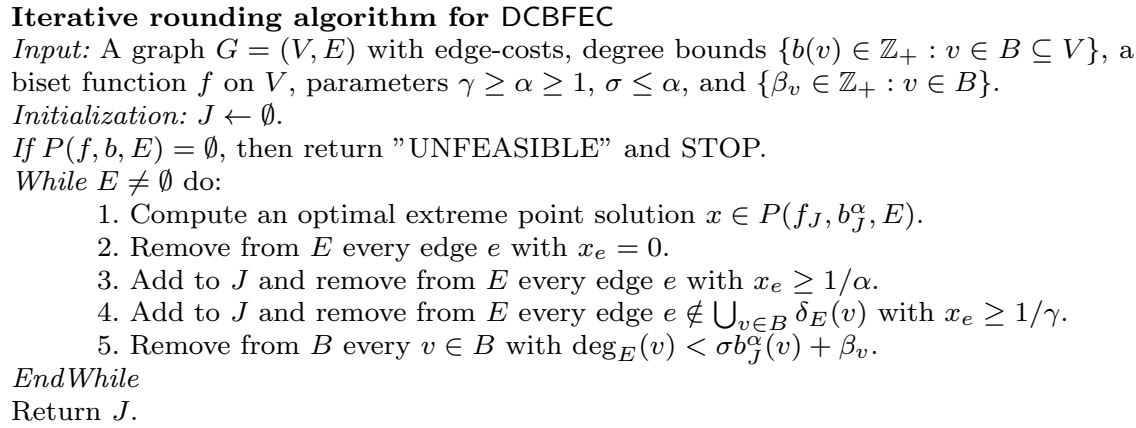

Fig. 1 Iterative rounding algorithm for DCBFEC problems.

\section{Iterative rounding for degree constrained connectivity problems}

Let us recall some facts from polyhedral theory. Let $\Pi \subseteq \mathbb{R}^{m}$ be a polytope defined by a system of linear inequalities and let $x \in \Pi$. $x$ is an extreme point of $\Pi$ if it is not a convex combination of other points in $\Pi$. $x$ is a basic feasible solution to the system that defines $\Pi$, if there exists a set of $m$ inequalities such that $x$ is the unique solution for the corresponding linear equations system; that is, the corresponding $m$ equations are linearly independent and each of them holds as equality for $x$. It is well known that $x$ is an extreme point of $\Pi$ if and only if $x$ is a basic feasible solution to the system that defines $\Pi$. It is also known that if the LP $\min \{c \cdot x: x \in \Pi\}$ has an optimal solution, then it has an optimal solution which is basic.

Given a biset function $f$ and an edge-set or a graph $J$, the residual biset function of $f$ is $f_{J}(\hat{S})=f(\hat{S})-\left|\delta_{J}^{i n}(\hat{S})\right|$. Given a parameter $\alpha \geq 1$, the residual degree bounds are $b_{J}^{\alpha}(v)=b(v)-\left|\delta_{J}(v)\right| / \alpha$. It is known that if $f$ admits a polynomial time evaluation oracle and is intersecting supermodular or is weakly supermodular, then so is $f_{J}$. Particular cases of the generic algorithm for DCBFEC in Figure 1 were used in various papers for set functions, c.f. [18, $2,19,23]$. The algorithm starts with $J=\emptyset$ and performs iterations. In every iteration, one considers the residual polytope $P\left(f_{J}, b_{J}^{\alpha}, E\right)$, and removes some edges from $E$ or some nodes from $B$, until $E$ becomes empty. The performance of the algorithm is summarized in the following statement, which proof is almost identical to the ones in $[18,2,19,23]$ for set functions.

Lemma 1 If the algorithm in Figure 1 terminates (and does not return " $U N$ FEASIBLE"), then it computes an $f$-connected subgraph $J$ of $G$ such that $c(J) \leq \gamma \tau$ and such that for every $v \in B$ the following holds:

- If $\sigma=0$ then $\operatorname{deg}_{J}(v) \leq \alpha b(v)$ or $\operatorname{deg}_{J}(v)<\alpha b(v)+\beta_{v}-1 ;$ thus $\operatorname{deg}_{J}(v) \leq$ $\lceil\alpha b(v)\rceil+\max \left\{\beta_{v}-2,0\right\}$.

- If $\sigma>0$ then $\operatorname{deg}_{J}(v)<\alpha b(v)+\beta_{v}$; thus $\operatorname{deg}_{J}(v) \leq\lceil\alpha b(v)\rceil+\beta_{v}-1$.

Proof The cost approximation proof is the same as in the paper of Jain [14]. We prove the approximation of the degrees. Consider a node $v \in B$. Let $J^{\prime}$ be 
the set of edges added to $\delta_{J}(v)$ while $v \in B$, and let $J^{\prime \prime}$ be the set of edges in $\delta_{E}(v)$ when $v$ was excluded from $B$. Note that $\left|J^{\prime}\right|=\alpha\left(b(v)-b_{J^{\prime}}^{\alpha}(v)\right)$. If $b_{J^{\prime}}^{\alpha}(v)=0$ then $J^{\prime \prime}=\emptyset$, and then $|J|=\left|J^{\prime}\right| \leq \alpha b(v)$. Suppose that $b_{J^{\prime}}^{\alpha}(v)>0$, so $\left|J^{\prime}\right|<\alpha b(v)$. If $\sigma=0$ then $\left|J^{\prime \prime}\right| \leq \beta_{v}-1$, hence $\operatorname{deg}_{J}(v)=\left|J^{\prime}\right|+\left|J^{\prime \prime}\right|<$ $\alpha b(v)+\beta_{v}-1$. If $\sigma>0$ then $\left|J^{\prime \prime}\right|<b_{J^{\prime}}^{\alpha}(v)+\beta_{v}$, hence $\operatorname{deg}_{J}(v)=\left|J^{\prime}\right|+\left|J^{\prime \prime}\right|<$ $\alpha\left(b(v)-b_{J^{\prime}}^{\alpha}(v)\right)+b_{J^{\prime}}^{\alpha}(v)+\beta_{v}<\alpha b(v)+\beta_{v}$.

We prove the following sequence of theorems, that imply Theorems $6,7,8$.

Theorem 9 (Implies Theorem 6) Let $x$ be an extreme point of $P(f, b, E)$ with $x_{e}>0$ for all $e \in E$, where $G=(V, E)$ is a directed graph, $f$ is an intersecting supermodular set function on $V$ and $\{b(v) \geq 0: v \in B \subseteq E\}$. Let $I$ be the set of edges in $E$ with tail in $B$ and let $F=E \backslash I$. Then for any $\varepsilon \in[0,1 / 2)$ at least one of the following two properties holds:

(P1) There is $e \in I$ such that $x_{e} \geq 1-\varepsilon$ or there is $e \in F$ such that $x_{e} \geq \varepsilon$.

(P2) There is $v \in B$ such that $\operatorname{deg}_{E}(v)<b(v)+4$.

Theorem 9 is proved in Section 4. Theorem 6 (and thus also Theorem 1) follows from Theorem 9 by substituting in Lemma $1 \alpha=\frac{1}{1-\varepsilon}, \gamma=\frac{1}{\varepsilon}, \sigma=1$, and $\beta_{v}=4$, which gives $\operatorname{deg}_{J}(v) \leq\lceil\alpha b(v)\rceil+\beta_{v}-1=\left\lceil\frac{b(v)}{1-\varepsilon}\right\rceil \cdot b(v)+3$.

Theorem 10 (Implies Theorem 7) Let $x$ be an extreme point of $P(f, b, E)$ with $x_{e}>0$ for all $e \in E$, where $G=(V, E)$ is a directed graph, $f$ is an intersecting supermodular biset function on $V$. Then there is $e \in E$ with $x_{e} \geq$ $1 / 2$ or there is $v \in B$ such that $\operatorname{deg}_{E}(v)<2 b(v)+\Delta_{f}(v)+2$. In the case of indegree bounds, for any extreme point $x \in P\left(f, b^{i n}, E\right)$ there is an edge $e \in E$ with $x_{e} \in\{0,1\}$.

Theorem 10 is proved in Section 6. Theorem 7 (and thus also Theorem 2) follows from Theorem 10. The case of outdegree constraints in Theorem 7 follows from Theorem 10 by substituting in Lemma $1 \alpha=\sigma=\gamma=2$, and $\beta_{v}=\Delta_{f}(v)+1$. Then Lemma 1 gives the degree bound $\operatorname{deg}_{J}(v) \leq$ $\lceil\alpha b(v)\rceil+\beta_{v}=2 b(v)+\Delta_{f}(v)+2$. The case of indegree bounds also follows from Theorem 10, while the undirected case is easily deduced from the directed one, see Section 6.3.

Theorem 11 (Implies Theorem 8) Let $x$ be an extreme point of $P(f, b, E)$ with $x_{e}>0$ for all $e \in E$, where $G=(V, E)$ is an undirected graph, $f$ is a weakly supermodular symmetric biset function on $V$, and $\{b(v) \geq 0: v \in B \subseteq$ $V\}$. Then there is $e \in E$ with $x_{e} \geq 1 / 3$ or there is $v \in B$ with $\operatorname{deg}_{E}(v)<$ $\max \left\{\Delta_{f}^{*}(v), 3\right\}+3$.

Theorem 11 is proved in Section 7. Theorem 8 (and thus also Theorem 3) follows from Theorem 11 by substituting in Lemma $1 \sigma=0, \alpha=\gamma=3$, and $\beta_{v}=\max \left\{\Delta_{f}^{*}(v), 3\right\}+3$ for all $v \in B$. Then Lemma 4 gives the degree bound $\operatorname{deg}_{J}(v) \leq\lceil\alpha b(v)\rceil+\max \left\{\beta_{v}-2,0\right\}=3 b(v)+\max \left\{\Delta_{f}^{*}(v), 3\right\}+1$.

Recall that a set family $\mathcal{L}$ is laminar if for any distinct sets $X, Y \in \mathcal{L}$ either $X \subset Y$, or $Y \subset X$, or $X \cap Y=\emptyset$. We extend this definition to biset-families in two ways. 
Definition 4 We say that a biset $\hat{Y}$ contains a biset $\hat{X}$ and write $\hat{X} \subseteq \hat{Y}$ if $X \subseteq Y$ and $X^{+} \subseteq Y^{+}$; if also $\hat{X} \neq \hat{Y}$ then $\hat{X} \subset \hat{Y}$ and $\hat{Y}$ properly contains $\hat{X}$. A biset family $\mathcal{L}$ is laminar if for any $\hat{X}, \hat{Y} \in \mathcal{L}$ either $\hat{X} \subseteq \hat{Y}$, or $\hat{Y} \subseteq \hat{X}$, or $\hat{X}, \hat{Y}$ are disjoint (namely, $X \cap Y=\emptyset$ ). A biset family $\mathcal{L}$ is strongly laminar if for any $\hat{X}, \hat{Y} \in \mathcal{L}$ either $\hat{X} \subseteq \hat{Y}$, or $\hat{Y} \subseteq \hat{X}$, or $\hat{X}, \hat{Y}$ are strongly disjoint (namely, $X \cap Y^{+}, Y \cap X^{+}=\emptyset$ ).

The following statement is proved using a standard "uncrossing" argument, c.f. $[7,4]$ where the case without degree constraints was considered; we provide a proof-sketch of one case for completeness of exposition.

Intuitively, "uncrossing" two bisets $\hat{X}, \hat{Y}$ means the following. In the case of intersecting supermodular $f, \hat{X}, \hat{Y}$ with $X \cap Y \neq \emptyset$ are replaced by $\hat{X} \cap \hat{Y}, \hat{X} \cup \hat{Y}$. Note that if $\{\hat{X} \cap \hat{Y}, \hat{X} \cup \hat{Y}\}=\{\hat{X}, \hat{Y}\}$ for all $\hat{X}, \hat{Y} \in \mathcal{F}$ with $X \cap Y \neq \emptyset$, then $\mathcal{F}$ must be laminar. In the case of a skew supermodular $f, \hat{X}, \hat{Y}$ are replaced either by $\hat{X} \cup \hat{Y}, \hat{X} \cup \hat{Y}$, or by $\hat{X} \backslash \hat{Y}, \hat{Y} \backslash \hat{X}$. Note that if $\{\hat{X} \cap \hat{Y}, \hat{X} \cup \hat{Y}\}=\{\hat{X}, \hat{Y}\}$ or $\{\hat{X} \backslash \hat{Y}, \hat{Y} \backslash \hat{Y}\}=\{\hat{X}, \hat{Y}\}$ for all $\hat{X}, \hat{Y} \in \mathcal{F}$, then $\mathcal{F}$ must be strongly laminar.

For a biset family $\mathcal{F}$ let $\chi_{E}(\mathcal{F})$ denote the set of the incidence vectors of the edge-sets in the family $\left\{\delta_{E}^{i n}(\hat{S}): \hat{S} \in \mathcal{F}\right\}$. Similarly, for $T \subseteq B, \chi_{E}(T)$ is the set of incidence vectors of the edge-sets in $\left\{\delta_{E}(v): v \in T\right\}$.

Lemma 2 Let $f$ be a biset function and $G$ a graph on $V$. Then for any extreme point $x$ of $P(f, b, E)$ with $0<x_{e}<1$ for all $e \in E$, there exist a family $\mathcal{L} \subseteq \mathcal{V}$ and $T \subseteq B$, such that $f(\hat{S}) \geq 1$ for all $\hat{S} \in \mathcal{L}$, and such that the vectors in $\chi_{E}(\mathcal{L}) \cup \chi_{E}(T)$ are linearly independent and $|\mathcal{L}|+|T|=|E|$. Furthermore, the following holds.

(i) If $f$ is intersecting supermodular and $G$ is directed then there exists such $\mathcal{L}$ that is laminar.

(ii) If $f$ is weakly supermodular and $G$ is undirected, then there exists such $\mathcal{L}$ that is strongly laminar.

Proof We provide a proof-sketch for the case of weakly supermodular $f$ and undirected $G$; the proof for the case of intersecting supermodular $f$ and directed $G$ is similar. Given a set $\chi$ of vectors let $\operatorname{span}(\chi)$ denote the linear space spanned by them. Fleischer, Jain, and Williamson [7] proved the following: Let $G=(V, E)$ be an undirected graph and $f$ a weakly supermodular biset function on $V$. Let $x \in \mathbb{R}_{+}^{E}$ and $\mathcal{F}=\left\{\hat{S} \in \mathcal{V}: x\left(\delta_{E}(\hat{S})\right)=f(\hat{S})\right\}$. If $\mathcal{L}^{\prime}$ is an inclusion maximal strongly laminar subfamily of $\mathcal{F}$ then $\operatorname{span}\left(\chi\left(\mathcal{L}^{\prime}\right)\right)=\operatorname{span}(\chi(\mathcal{F}))$.

Let $\mathcal{F}$ and $\mathcal{L}^{\prime}$ be as in the above result of [7]. Let $\mathcal{L}$ be an inclusion maximal sub-family of $\mathcal{L}^{\prime}$ such that the vectors in $\chi(\mathcal{L})$ are linearly independent, so $\operatorname{span}(\chi(\mathcal{L}))=\operatorname{span}\left(\chi\left(\mathcal{L}^{\prime}\right)\right)=\operatorname{span}(\chi(\mathcal{F}))$. Let $T$ be a maximal subset of $Q=\left\{v \in B: x\left(\delta_{E}(v)\right)=b(v)\right\}$ such that the vectors in $\chi(\mathcal{L}) \cup \chi(T)$ are linearly independent. Note that $\operatorname{span}(\chi(\mathcal{L}) \cup \chi(T))=\operatorname{span}(\chi(\mathcal{F}) \cup \chi(Q))$. Since $x$ is an extreme point, the dimension of the vector space $\operatorname{span}(\chi(\mathcal{F}) \cup \chi(Q))$ is exactly $|E|$, which implies $|\mathcal{L}|+|T|=|\chi(\mathcal{L})|+|\chi(T)|=|E|$.

Any laminar biset family $\mathcal{L}$ defines a tree order on its members by the inclusion as in Definition 4; this order coincides with the tree order defined by 
an ordinary inclusion of the inner parts of the bisets in $\mathcal{L}$. We carry the usual notion of children, descendants, ancestors, and leaf-sets, from laminar families of sets to laminar families of bisets. Given a biset $\hat{S} \in \mathcal{L}$ and an edge-set $E$ let us use the following notation.

- $E_{S}^{+}$is the set of edges in $E$ covering $\hat{S}$ but not a child of $\hat{S}$.

- $E_{S}^{-}$is the set of edges in $E$ covering some child of $\hat{S}$ but not $\hat{S}$.

- $E_{S}=E_{S}^{+} \cup E_{S}^{-}$is the set of edges in $E$ covering either $\hat{S}$ or a child of $\hat{S}$.

Lemma 3 Let $G=(V, E)$ be a graph with edge weights $\left\{x_{e}: e \in E\right\}$ and let $\mathcal{L}$ be a laminar biset family on $V$. Then the following holds for every $\hat{S} \in \mathcal{L}$ if $G$ is directed, and for every $\hat{S} \in \mathcal{L}^{1}$ if $G$ is undirected, where $\mathcal{C}$ is the is the set of children in $\mathcal{L}$ of $\hat{S}$ :

$$
x\left(E_{S}^{+}\right)-x\left(E_{S}^{-}\right)=x\left(\delta_{E}^{i n}(\hat{S})\right)-\sum_{C \in \mathcal{C}} x\left(\delta_{E}^{i n}(\hat{C})\right) .
$$

Proof The statement is easily verified by counting the contribution of every edge in $E_{S}$ to each side.

Corollary 2 In the setting of Lemma 3, suppose that $x\left(\delta_{E}^{i n}(\hat{Y})\right)$ is a positive integer for every $\hat{Y} \in\{\hat{S}\} \cup \mathcal{C}, \delta_{E}^{i n}(\hat{S}) \neq \bigcup_{C \in \mathcal{C}} \delta_{E}^{i n}(\hat{C})$, and $0<x_{e} \leq 1 /(\alpha-\epsilon)$ for all $e \in E_{S}$, where $\alpha \geq 1$ and $0<\epsilon<\alpha$. If one of the edge sets $E_{S}^{+}, E_{S}^{-}$is empty, then the other has at least $\lceil\alpha+\epsilon\rceil$ edges. In particular, $\left|E_{S}\right| \geq 2$.

Proof Suppose that $E_{S}^{-}=\emptyset$. Then $E_{S}^{+} \neq \emptyset$, as otherwise $\delta_{E}^{i n}(\hat{S})=\bigcup_{C \in \mathcal{C}} \delta_{E}^{i n}(\hat{C})$. By Lemma $3, x\left(E_{S}^{+}\right)$is an integer, and it must be a positive integer, since $E_{S}^{+} \neq \emptyset$ and since $x_{e}>0$ for all $e \in E_{S}$. Since $0<x_{e}<1 /(\alpha+\epsilon)$ for all $e \in E_{S}$, we get that $\left|E_{S}^{+}\right| \geq\left\lceil(\alpha+\epsilon) x\left(E_{S}^{+}\right)\right\rceil \geq\lceil\alpha+\epsilon\rceil$.

The proof of the case $E_{S}^{+}=\emptyset$ is similar.

\section{Proof of Theorem 9}

The following lemma is used in the proof of Theorems 9 and 10 .

Lemma 4 Let $x$ and $\mathcal{L}$ be as in Lemma 2, where $G$ is a directed graph and $\mathcal{L}$ is a laminar set family, and let $\left\{\beta_{v} \in \mathbb{Z}_{+}: v \in B\right\}$. Let $I$ be the set of edges in $E$ with tail in $B$ and let $F=E \backslash I$. Then there is $v \in B$ such that $\operatorname{deg}_{E}(v)<\sigma b(v)+\beta_{v}$, if for some $\theta>0$ the following inequality holds:

$$
|\mathcal{L}|<(\theta \beta(B)-|B|)+(1-\theta)|I|+\theta \sigma x(I)+|F| .
$$

Proof Note that since $x\left(\delta_{E}(v)\right) \leq b(v)$ and $\delta_{E}(v)=\delta_{I}(v)$ for all $v \in B$ then

$$
\sum_{v \in B}\left(\operatorname{deg}_{E}(v)-\sigma b(v)\right) \leq \sum_{v \in B}\left(\operatorname{deg}_{E}(v)-\sigma x\left(\delta_{E}(v)\right)\right)=|I|-\sigma x(I) .
$$

Thus $|I|-\sigma x(I)<\sum_{v \in B} \beta_{v}=\beta(B)$ implies that $\operatorname{deg}_{E}(v)<b(v)+\beta_{v}$ for some $v \in B$. Adding $|F|$ to both sides of the inequality $|I|-\sigma x(I)<\beta(B)$ gives 
$|I|+|F|<\beta(B)+\sigma x(I)+|F|$. Note that since $|I|+|F|=|E|=|\mathcal{L}|+|T|$, we have $|I|+|F|=\frac{1}{\theta}(|\mathcal{L}|+|T|)+\left(1-\frac{1}{\theta}\right)(|I|+|F|)$. Consequently, since $|T| \leq|B|$, to prove that there is $v \in B$ such that $\operatorname{deg}_{E}(v)<\alpha b(v)+\beta_{v}$, it is sufficient to prove that

$$
\frac{1}{\theta}(|\mathcal{L}|+|B|)+\left(1-\frac{1}{\theta}\right)(|I|+|F|)<\beta(B)+\sigma x(I)+|F| .
$$

Multiplying both sides by $\theta$ and rearranging terms gives (3).

Now we prove Theorem 9. We will assume that Property (P1) does not hold, namely, that

$$
x_{e}<1-\varepsilon \text { for all } e \in I \text { and } x_{e}<\varepsilon \text { for all } e \in F
$$

and prove that Property (P2) must hold. Substituting in (3) $\theta=1 / 2, \sigma=1$, and $\beta_{v}=4$ for all $v \in B$, gives

$$
|\mathcal{L}|<|B|+\frac{1}{2}|I|+\frac{1}{2} x(I)+|F| .
$$

To show that the latter inequality holds, we assign tokens to edges in $E$ and nodes in $B$ of total amount that equals the right-hand side, as follows.

- 1 token to every $v \in B$, placed at $v$.

- $\frac{1}{2}\left(1+x_{e}\right)$ "head-tokens" to every $e \in E$ placed at the head of $e$.

- $\frac{1}{2}\left(1-x_{e}\right)$ additional "tail-tokens" to every $e \in F$ placed at the tail of $e$.

We will show that assumption (4) implies that these tokens can be redistributed among the sets in $\mathcal{L}$ such that every set gets at least 1 token, and some spare tokens remain. Let us say that a leaf-set $S \in \mathcal{L}^{0}$ is dangerous if $S \cap B=\emptyset$ and $\left|\delta_{E}^{i n}(S)\right|=2$. We prove the following.

Lemma 5 For any $S \in \mathcal{L}$ it is possible to redistribute the $S$-tokens such that every proper descendant of $S$ in $\mathcal{L}$ gets 1 token, and such that: $S$ gets $3 / 2$ tokens if $S$ is dangerous, and $S$ gets 2 tokens otherwise.

Proof The proof is by induction on the number of descendants of $S$ in $\mathcal{L}$. If $S$ is a leaf set, then $S$ gets $\left(\left|E_{S}^{+}\right|+x\left(E_{S}^{+}\right)\right) / 2 \geq\left(\left|E_{S}^{+}\right|+1\right) / 2$ head tokens; this is at least $3 / 2$, and is at least 2 if $S$ is dangerous.

Suppose that $S$ is not a leaf. Let $t=x\left(E_{S}^{+}\right)-x\left(E_{S}^{-}\right)+\left|E_{S}^{-}\right|$. By Lemma 3 , $t=f(S)-\sum_{C \in \mathcal{C}} f(C)+\left|E_{S}^{-}\right|$, where $\mathcal{C}$ is the set of children of $S$; hence $t$ is an integer. Since $\left|E_{S}^{-}\right| \geq x\left(E_{S}^{-}\right), t \geq 1$. The amount of tokens $S$ gets from edges in $E_{S}$ is $\left(\left|E_{S}^{+}\right|+x\left(E_{S}^{+}\right)\right) / 2+\left(\left|E_{S}^{-}\right|-x\left(E_{S}^{-}\right)\right) / 2=\left(t+\left|E_{S}^{+}\right|\right) / 2$. Consequently, $S$ can collect 2 tokens from its children, edges in $E_{S}$, and nodes in $B$, unless $S$ owns no node in $B$, and either (see Figure 2):

(a) $S$ has two children, both dangerous, and $E_{S}^{+}=\emptyset$ (see Figure 2(a)).

(b) $S$ has 1 dangerous child and $\left|E_{S}^{+}\right| \leq 1$ (see Figure 2(b)).

(c) $S$ has 1 non dangerous child and $E_{S}^{+}=\emptyset$ (see Figure 2(c)). 
(a)

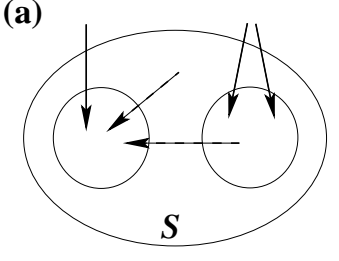

(b)

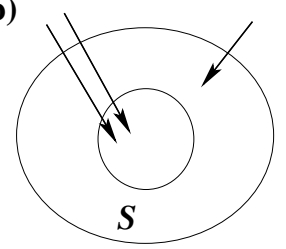

(c)

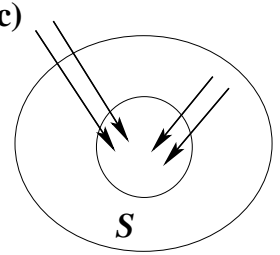

Fig. 2 Illustration to the proof of Lemma 5.

Case (a) is not possible. To see this, note that by (4), the two edges entering a dangerous set belong to $I$, and thus have tail in $B$. Hence the tail of an edge that enters a child of $S$ cannot be in $S$; namely, the dashed edges in figure 2(a) do not exist. Consequently, every edge that enters a child of $S$ also enters $S$. Since $E_{S}^{+}=\emptyset$ we get that the edges entering $S$ is a disjoint union of the edges entering the children of $S$. This contradicts linear independence.

Case (b) is not possible by a similar argument. The tail of an edge that enters a child of $S$ cannot be in $S$, hence we must have $E_{S}^{-}=\emptyset$ in this case. This contradicts Corollary 2.

In Case (c), by a similar argument, we must have $\left|E_{S}^{-}\right| \geq 2$, which implies $t \geq\left|E_{S}^{-}\right| / 2 \geq 1$. In this case $S$ gets 1 token from edges in $E_{S}$ and one token from its non-dangerous child.

\section{Sharing property of laminar biset-families}

Given a laminar biset family $\mathcal{L}$ let $\mathcal{L}^{p}$ denote the members of $\mathcal{L}$ that have exactly $p$ children in $\mathcal{L}$; in particular, $\mathcal{L}^{0}$ is the family of leaf-bisets of $\mathcal{L}$, and $\mathcal{L}^{1}$ is the family of bisets in $\mathcal{L}$ that have a unique child in $\mathcal{L}$. Note that

$$
|\mathcal{L}|<2\left|\mathcal{L}^{0}\right|+\left|\mathcal{L}^{1}\right|
$$

We now illustrate a key difficulty in applying token counting arguments in the case of laminar biset families. The token distribution schemes for set-functions (e.g., as in the proof of Theorem 9) rely on the fact that if $\mathcal{L}$ is a laminar set family, then the following holds.

(i) A set $S \in \mathcal{L}$ owns $v$ if, and only if, there can be an edge incident to $v$ that covers $S$ or a child of $S$, but no edge incident to $v$ can cover both.

(ii) For every $v \in V$ there is at most one set in $\mathcal{L}$ that owns $v$.

Now let $\mathcal{L}$ be a laminar biset family. For our purposes, it is sufficient to consider bisets in $\mathcal{L}^{0} \cup \mathcal{L}^{1}$ only, but even in this case, we cannot satisfy properties (i),(ii) simultaneously. Consider a biset $\hat{S} \in \mathcal{L}^{1}$ and its child $\hat{C}$. It is not hard to verify (see Figure 3 ) that for both directed and undirected graphs, $v$ satisfies property (i) if and only if $v$ belongs to the set $(S \backslash C) \cup\left(\Gamma(\hat{S}) \backslash C^{+}\right)$. These sets may not be pairwise disjoint, as for distinct $\hat{X}, \hat{Y} \in \mathcal{L}^{1}$ we may have that $\Gamma(\hat{X}) \backslash C_{X}^{+}$and $\Gamma(\hat{Y}) \backslash C_{Y}^{+}$intersect, where $\hat{C}_{X}$ is the child of $\hat{X}$ and $C_{Y}$ is the child of $\hat{Y}$. This motivates the following definition (see Figure 3 ). 

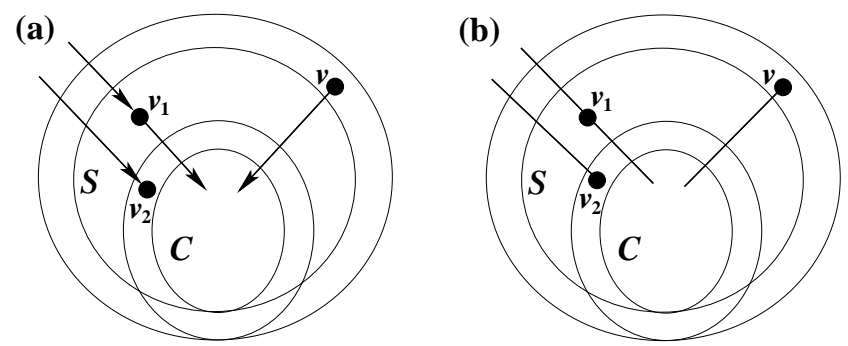

Fig. 3 Illustration to Definition 5 for $\hat{S} \in \mathcal{L}^{1}$ with child $\hat{C}$; $\hat{S}$ owns each of $v_{1}, v_{2}$ and shares $v$ (an edge in $\delta_{E_{S}}(v)$ must cover $\hat{C}$ ). (a) Undirected graphs. (b) Directed graphs.

Definition 5 Let $\mathcal{L}$ be a laminar biset family on $V$, let $\hat{S} \in \mathcal{L}^{0} \cup \mathcal{L}^{1}$, and let $\hat{C}$ be the child of $\hat{S}$ if $\hat{S} \in \mathcal{L}^{1}$ and $\hat{C}=(\emptyset, \emptyset)$ if $\hat{S} \in \mathcal{L}^{0}$. We say that $\hat{S}$ owns $v \in V$ if $v \in S \backslash C$, and for $\hat{S} \in \mathcal{L}^{1}$ we say that $\hat{S}$ shares $v$ if $v \in \Gamma(\hat{S}) \backslash C^{+}$. Let $\mathcal{L}^{1}(v)$ denote the family of bisets in $\mathcal{L}^{1}$ sharing $v$.

The following two statement are easily verified.

Lemma 6 Let $G=(V, E)$ be a graph, let $\mathcal{L}$ be a laminar biset family on $V$, let $\hat{S} \in \mathcal{L}^{0} \cup \mathcal{L}^{1}$, and let $e \in E_{S}$ (for illustration see Figure 3).

(i) If $G$ is a directed graph then the following holds. If $e \in E_{S}^{+}$then $\hat{S}$ owns the head of $e$, and if $e \in E_{S}^{-}$then $\hat{S}$ owns or shares the tail of $e$.

(ii) If $G$ is an undirected graph then the following holds. If $e \in E_{S}^{+}$then $\hat{S}$ owns one endnode of $e$, and if $e \in E_{S}^{-}$then $\hat{S}$ owns or shares one endnode of $e$.

Lemma 7 Let $\mathcal{L}$ be a laminar biset family on $V$ and let $v \in V$.

(i) There is at most one biset that owns $v$.

(ii) If two bisets share $v$ then none of them is a descendant of the other.

By the definition of the parameters $\Delta_{f}(v), \Delta_{f}^{*}(v)$ and part (ii) of the last lemma, we have the following.

Corollary 3 Let $f$ and $\mathcal{L}$ be as in Lemma 2. Then $\left|\mathcal{L}^{1}(v)\right| \leq \Delta_{f}(v)$, and if $\mathcal{L}$ is strongly laminar then $\left|\mathcal{L}^{1}(v)\right| \leq \Delta_{f}^{*}(v)$.

Lemma 8 Let $\mathcal{L}$ be a laminar biset family on $V$, let $G=(V, E)$ be a directed/undirected graph, let $v \in V$, and let $\hat{S}, \hat{X} \in \mathcal{L}^{0} \cup \mathcal{L}^{1}$ be distinct. Then $\delta_{E_{S}}(v) \cap \delta_{E_{X}}(v)=\emptyset$ if both $\hat{S}, \hat{X}$ share $v$, or if $\hat{S}$ shares $v$ and $\hat{X}$ owns $v$ and one of the following holds:

(i) $G$ is a directed graph.

(ii) $G$ is an undirected graph and $\mathcal{L}$ is strongly laminar.

Proof Let $\hat{C}_{S}$ be the child of $\hat{S}$ and $\hat{C}_{X}$ the child of $\hat{X}$, if such exists. Let $e_{S} \in \delta_{E_{S}}(v)$ and $e_{X} \in \delta_{E_{X}}(v)$. We claim that $e_{S} \neq e_{X}$ in the cases of the lemma. If $\hat{S}, \hat{X}$ share $v$, then $e_{S}$ covers $\hat{C}_{S}$ and $e_{X}$ covers $\hat{C}_{X}$ (see Figure 3 ). By Lemma 7 (ii), $C_{S} \cap C_{X}=\emptyset$, hence $e_{S} \neq e_{X}$, as required. 
(a)

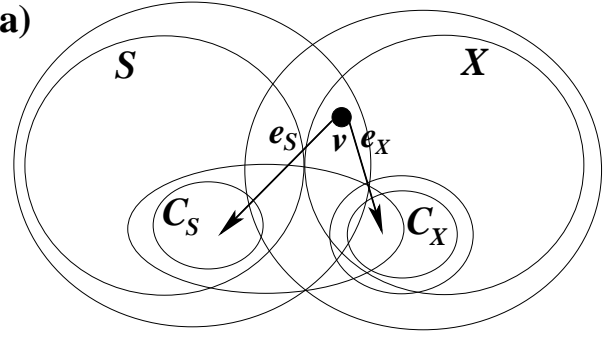

(b)

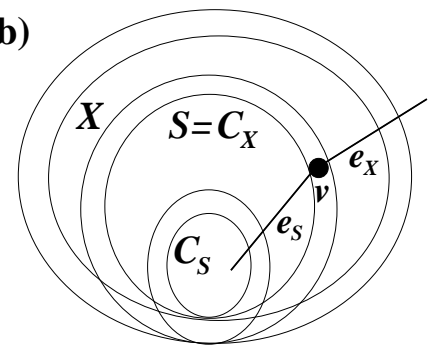

Fig. 4 Illustration to the proof of Lemma 8 for the case when $\hat{S}$ shares $v$ and $\hat{X}$ owns $v$.

Suppose that $\hat{S}$ shares $v$ and $\hat{X}$ owns $v$. Assume that none of $\hat{X}, \hat{S}$ contains the other (see Figure $4(\mathrm{a})$ ). Since $v \in X \cap \Gamma(\hat{S}), \mathcal{L}$ cannot be strongly laminar. Hence the relevant case is when $G$ is a directed graph. By the definition of the sets $E_{S}, E_{X}, e_{S}$ has its head in $C_{S}$ and $e_{X}$ has its head in $C_{X}$. Since $\mathcal{L}$ is laminar, $C_{S} \cap C_{X}=\emptyset$. This implies $e_{S} \neq e_{X}$. Now assume that one of $\hat{X}, \hat{S}$ contains the other. Since $v \in X \cap \Gamma(\hat{S})$, we must have $\hat{S} \subseteq \hat{X}$ (possibly $\hat{S}=\hat{C}_{X}$, which is the case depicted in Figure 4(a)). Since $\hat{S} \subseteq \hat{X}$ and $v \in X \cap \Gamma(\hat{S})$, $e_{X}$ cannot cover $\hat{C}_{X}$. Hence $e_{X}$ covers $\hat{X}$ and the relevant case is when $G$ is an undirected graph. On the other hand, $e_{S}$ covers $\hat{C}_{S}$, since $v \in \Gamma(\hat{S})$. Summarizing, $e_{X}$ goes from $v$ to $V \backslash X^{+}$and $e_{S}$ goes from $v$ to $C_{S}$. Since $\left(V \backslash X^{+}\right) \cap C_{S}=\emptyset$, we must have $e_{S} \neq e_{X}$.

\section{Proof of Theorem 10}

\subsection{Directed graphs and outdegree constraints}

Let $x, \mathcal{L}$ be as in Lemma 2, and let $I, F$ be as in Lemma 4. Assume that $0<x_{e}<1 / 2$ for all $e \in E$. Substituting in (3) $\theta=1, \sigma=2$, and $\beta_{v}=$ $\Delta_{\mathcal{L}^{1}}(v)+2 \leq \Delta_{f}(v)+2$ for all $v \in B$, gives

$$
|\mathcal{L}|<\Delta_{\mathcal{L}^{1}}(B)+|B|+2 x(I)+|F| .
$$

To show that the latter inequality holds, we assign tokens to edges in $E$ and nodes in $B$ of total amount at most the right-hand side, as follows.

- $\Delta_{\mathcal{L}^{1}}(v)+1$ "shared-tokens" to every $v \in B$.

- $2 x_{e}$ "head-tokens" to every $e \in E$ placed at the head of $e$.

- $1-2 x_{e}$ additional "tail-tokens" to every $e \in F$ placed at the tail of $e$.

By (5) we have $|\mathcal{L}|<2\left|\mathcal{L}^{0}\right|+\left|\mathcal{L}^{1}\right|$, hence it is sufficient to prove the following.

Lemma 9 We can reassign the tokens to the members of $\mathcal{L}^{0} \cup \mathcal{L}^{1}$ such that every $\hat{S} \in \mathcal{L}^{0}$ gets at least 2 tokens and every $\hat{S} \in \mathcal{L}^{1}$ gets at least 1 token.

Proof Token reassignment rules are as follows. 
- If $\hat{S} \in \mathcal{L}^{1}$ owns or shares $v \in B$ then $\hat{S}$ gets 1 shared-token from $v$.

- If $\hat{S} \in \mathcal{L}^{0} \cup \mathcal{L}^{1}$ then $\hat{S}$ gets the tail-tokens from each edge in $E_{S}^{-}$and the head-tokens from each edge in $E_{S}^{+}$.

Since by Lemma 7(i) every node is owned by at most one biset, the total amount of tokens a node $v \in B$ gives to bisets does not exceed the amount of tokens initially placed at $v$. Also, from Lemma 8 and Lemma 6 , it follows that for every $e \in E$, the tail-tokens of $e$ are assigned to at most one biset, and the head-tokens of $e$ are assigned to at most one biset.

If $\hat{S} \in \mathcal{L}^{0}$ is a leaf set then $\hat{S}$ gets from edges in $\delta_{E}^{i n}(\hat{S})$ exactly $2 x\left(\delta_{E}^{i n}(\hat{S})\right)=$ $2 f(\hat{S}) \geq 2$ head-tokens. Let $\hat{S} \in \mathcal{L}^{1}$ and let $\hat{C}$ be the child of $\hat{S}$. If the tail $v$ of some edge in $E_{S}^{-}$is in $B$, then $\hat{S}$ owns or shares $v$, by Lemma 6 . In this case $\hat{S}$ gets 1 token from $v$. Otherwise, all edges in $E_{S}^{-}$have their tail in $V \backslash B$, so $E_{S}^{-} \subseteq F$; thus $\hat{S}$ gets $\left|E_{S}^{-}\right|-2 x\left(E_{S}^{-}\right)$tail-tokens from edges in $\left|E_{S}^{-}\right|$. In addition, $\hat{S}$ also gets $2 x\left(E_{S}^{+}\right)$head tokens from edges in $E_{S}^{+}$. Thus $\hat{S}$ gets $2 x\left(E_{S}^{+}\right)-2 x\left(E_{S}^{-}\right)+\left|E_{S}^{-}\right|=2\left(x\left(E_{S}^{+}\right)-x\left(E_{S}^{-}\right)\right)+\left|E_{S}^{-}\right|$tokens from edges in $E_{S}$. By Lemma 3 , this is an integer, and it must be a positive integer, since $x_{e}<\frac{1}{2}$ for all $e \in E$ implies $\left|E_{S}^{-}\right|-2 x\left(E_{S}^{-}\right)>0$.

\subsection{Directed graphs and indegree constraints}

Suppose to the contrary that $0<x_{e}<1$ for all $e \in E$. Then similarly to Lemma 2 we have the following. There exist a laminar family $\mathcal{L}$ on $V$ and $T^{\text {in }} \subseteq B$, such that $f(\hat{S}) \geq 1$ for all $\hat{S} \in \mathcal{L}$, and such that the vectors in $\chi_{E}(\mathcal{L}) \cup \chi_{E}\left(T^{i n}\right)$ are linearly independent and $|\mathcal{L}|+\left|T^{i n}\right|=|E|$. Now let us consider the biset family $\mathcal{F}=\mathcal{L} \cup\left\{(v, v): v \in T^{i n}\right\}$. Then $|\mathcal{F}|=|E|$ and the characteristic vectors of the edge sets $\left\{\delta_{E}^{i n}(\hat{S}): S \in \mathcal{F}\right\}$ are linearly independent. Let $\mathcal{F}_{\max }$ denote the family of the inclusion-maximal members of $\mathcal{L}$; note that $\left|\mathcal{F}_{\max }\right| \geq 1$. To obtain a contradiction, we prove that $2|E|-$ $\left|\mathcal{F}_{\max }\right| \geq 2|\mathcal{F}|$. We will assign 2 tokens to every $e \in E$ as follows.

- 1 "head-token" placed at the head of $e$.

- 1 "tail-token" placed at the tail of $e$.

The total amount of tokens is $2|E|$. We will show how to reassign $2|E|-\left|\mathcal{F}_{\max }\right|$ of these tokens to the members of $\mathcal{F}$, such that every member of $\mathcal{F}$ gets at least 2 tokens. The tokens reassignment rule is: every $\hat{S} \in \mathcal{F}$ gets the tailtokens of the edges in $E_{S}^{-}$and the head-tokens from edges in $E_{S}^{+}$. Note that the tail tokens of the edges entering the members of $\mathcal{F}_{\max }$ are not assigned. No head token is assigned twice by Lemma 6 , and no tail token is assigned twice by Lemma 8 , hence every token is assigned to at most one biset in $\mathcal{F}$. Every member of $\mathcal{F}$ gets at least 2 tokens, since $\left|E_{S}\right| \geq 2$; to see this, note that if one of $E_{S}^{+}, E_{S}^{-}$is empty then the other has at least 2 edges, by Corollary 2 and the assumption $x_{e}<1$ for all $e \in E$. 


\subsection{Undirected graphs}

In Theorem 7, the part concerning undirected graphs follows from the part concerning directed graphs, and the following (essentially known) statement; we provide a proof-sketch for completeness of exposition.

Lemma 10 Let $f$ be an intersecting supermodular biset function. Then any inclusion-minimal $f$-connected directed graph $J$ has maximum indegree at most $k=\max \{f(\hat{S}): \hat{S} \in \mathcal{V}\}$.

Proof Consider the biset family $\mathcal{F}=\left\{\hat{S}:\left|\delta_{J}^{i n}(S)\right|=f(S)\right\}$. We claim that $\mathcal{F}$ is an intersecting biset family, namely, for any bisets $\hat{X}, \hat{Y} \in \mathcal{F}$ which inner parts intersect, $\hat{X} \cap \hat{Y}, \hat{X} \cup \hat{Y} \in \mathcal{F}$. To see this, note that the biset function $\left|\delta_{J}^{i n}(\hat{S})\right|$ is submodular, and hence

$f(\hat{X})+f(\hat{Y})=\left|\delta_{J}^{i n}(\hat{X})\right|+\left|\delta_{J}^{i n}(\hat{Y})\right| \geq\left|\delta_{J}^{i n}(\hat{X} \cap \hat{Y})\right|+\left|\delta_{J}^{i n}(\hat{X} \cup \hat{Y})\right| \geq f(\hat{X} \cap \hat{Y})+f(\hat{X} \cup \hat{Y})$.

Since $f$ is intersecting supermodular, equality must hold everywhere, and thus $\left|\delta_{J}^{i n}(\hat{X} \cap \hat{Y})\right|=f(\hat{X} \cap \hat{Y})$ and $\left|\delta_{J}^{i n}(\hat{X} \cup \hat{Y})\right|=f(\hat{X} \cup \hat{Y})$, so $\hat{X} \cap \hat{Y}, \hat{X} \cup \hat{Y} \in \mathcal{F}$.

For an edge $e \in J$ let $\mathcal{F}_{e}=\left\{\hat{S} \in \mathcal{F}: e \in \delta_{J}^{i n}(\hat{S})\right\}$. Since $J$ is minimally $f$ connected, $\mathcal{F}_{e}$ is non-empty for every $e \in J$. Furthermore, $\mathcal{F}_{e}$ is an intersecting biset family, hence among all bisets $\hat{S} \in \mathcal{F}_{e}$ there is a unique biset $\hat{S}_{e}=$ $\left(S_{e}, S_{e}^{+}\right)$with $\left|S_{e}\right|+\left|S_{e}^{+}\right|$minimal.

Suppose to the contrary that $\operatorname{deg}_{J}(v) \geq k+1$ for some $v \in V$. Let $e \in \delta_{J}^{i n}(v)$ and let $\hat{S}_{e}$ be as above. Since $\operatorname{deg}_{H}(v) \geq k+1$ there is $e^{\prime} \in \delta_{J}^{i n}(v)$ that does not enter $\hat{S}_{e}$. Now consider the bisets $\hat{S}_{e}$ and $\hat{S}_{e^{\prime}}$. Their inner parts intersect (both contain $v$ ), hence $\hat{S}=\hat{S}_{e} \cap \hat{S}_{e^{\prime}} \in \mathcal{F}$. It is also easy to see that $e, e^{\prime} \in \delta_{J}^{i n}(\hat{S})$. This contradicts the minimality of $\left|S_{e}\right|+\left|S_{e}^{+}\right|$or of $\left|S_{e^{\prime}}\right|+\left|S_{e^{\prime}}^{+}\right|$.

Now we use the following standard reduction, that implies the undirected graphs part in Theorem 7 .

Proposition 1 For DCBFEC with intersecting supermodular biset function $f$, existence of a $(\rho(b(v)), \alpha)$-approximation algorithm for directed graphs implies existence of $a(\rho(b(v))+k, 2 \alpha)$-approximation algorithm for undirected graphs.

Proof Given an instance of undirected DCBFEC obtain an instance of directed DCBFEC by bidirecting the edges of $G$, namely, replacing every undirected edge $e=u v$ of $G$ by the two opposite directed edges $u v, v u$ of the same cost as $e$. Then apply the $(\rho(b(v)), \alpha)$-approximation algorithm on the obtained directed graph to compute a directed inclusion minimal $f$-connected subgraph $J^{\prime}$, and output the underlying graph $J$ of $J^{\prime}$. The biderection of any $f$-connected subgraph is also $f$-connected, and the outdegree of every node in the biderection equals the degree of this node in the original graph. As in any inclusion minimal $f$-connected directed graph the indegree of every node is at most $k$, in the underlying graph of $J^{\prime}$ the degree of every node is at most $\rho(b(v))+k$. It also a routine to show that the cost approximation is $2 \alpha$. 


\section{Proof of Theorem 11}

Assume to the contrary that $x_{e}<1 / 3$ for all $e \in E$ and that

$$
\operatorname{deg}_{E}(v) \geq \max \left\{\Delta_{f}^{*}(v)+3,6\right\} \quad \forall v \in B .
$$

Let $\mathcal{L}, T$ be as in Lemma 2, where $\mathcal{L}$ is strongly laminar. We obtain the contradiction $2|E| \geq 4\left|\mathcal{L}^{0}\right|+2\left|\mathcal{L}^{1}\right|+2|T|>2(|\mathcal{L}|+|T|)$ as follows. We will assign two tokens to every edge $e \in E$, placing one token at each of the endnodes of $e$. We then reassign these tokens such that every biset in $\mathcal{L}^{0}$ gets 4 tokens, every biset in $\mathcal{L}^{1}$ gets 2 tokens, and such that for every $v \in T$ at least 2 tokens placed at $v$ are not assigned.

Definition 6 Let $\hat{S} \in \mathcal{L}^{0} \cup \mathcal{L}^{1}$ and let $\hat{C}$ is the unique child of $\hat{S}$ if $\hat{S} \in \mathcal{L}^{1}$ and $\hat{C}=(\emptyset, \emptyset)$ if $\hat{S} \in \mathcal{L}^{0}$. The contribution $a_{v}(\hat{S})$ of $v \in V$ to $\hat{S}$ is the amount of tokens placed at $v$ that are assigned to $\hat{S}$, and it is defined by

$$
\begin{aligned}
& a_{v}(\hat{S})=\min \left\{\operatorname{deg}_{E}(v), 4\right\} \quad \text { if } v \in S \backslash C^{+} \\
& a_{v}(\hat{S})=1 \quad \text { if } v \in \Gamma(\hat{S}) \cup \Gamma(\hat{C}) \text { and } 1 \leq \operatorname{deg}_{E_{S}}(v) \leq 3 \\
& a_{v}(\hat{S})=2 \quad \text { if } v \in \Gamma(\hat{S}) \cup \Gamma(\hat{C}) \text { and } \operatorname{deg}_{E_{S}}(v) \geq 4 \\
& a_{v}(\hat{S})=0 \quad \text { otherwise }
\end{aligned}
$$

Let $a(\hat{S})=\sum_{v \in V} a_{v}(\hat{S})$ be the total amount of tokens assigned to $\hat{S}$.

Note that if $v$ is not owned or shared by $\hat{S}$ then $a_{v}(\hat{S})=0$. Denote by $A_{v}=\sum_{\hat{S} \in \mathcal{L}^{0} \cup \mathcal{L}^{1}} a_{v}(\hat{S})$ be the total amount of tokens that $v$ contributes to the members of $\mathcal{L}^{0} \cup \mathcal{L}^{1}$, and let $A=\sum_{v \in V} A_{v}=\sum_{\hat{S} \in \mathcal{L}^{0} \cup \mathcal{L}^{1}} a(\hat{S})$ be the total amount of tokens assigned to the members of $\mathcal{L}^{0} \cup \mathcal{L}^{1}$. We will show that $2|E|-2|T| \geq A \geq 4\left|\mathcal{L}^{0}\right|+2\left|\mathcal{L}^{1}\right|$.

Lemma $11 a(\hat{S}) \geq 4$ if $\hat{S} \in \mathcal{L}^{0}$ and $a(\hat{S}) \geq 2$ if $\hat{S} \in \mathcal{L}^{1}$.

Proof Let $\hat{S} \in \mathcal{L}^{0}$. By $(6), a(\hat{S}) \geq 4$ if $S \cap T \neq \emptyset$. Otherwise, the assumption " $x_{e}<1 / 3$ for all $e \in E$ " implies that $\left|\delta_{E}^{i n}(S)\right| \geq 4$, hence $a(\hat{S}) \geq\left|\delta_{E}^{i n}(S)\right| \geq 4$.

Let $\hat{S} \in \mathcal{L}^{1}$. Consider the set $V_{S}$ of the endnodes of the edges in $E_{S}$ that $\hat{S}$ owns or shares. We have $a_{v}(\hat{S}) \geq 1$ for every $v \in V_{S}$, by the definition, hence if $\left|V_{S}\right| \geq 2$ then we are done. Suppose therefore that $V_{S}=\{v\}$. Let $\hat{C}$ be the child of $\hat{S}$. By Corollary 2, $\left|E_{S}\right| \geq 2$, and if one of $E_{S}^{+}, E_{S}^{-}$is empty then $\left|E_{S}\right| \geq 4$. If $v \in S \backslash C^{+}$then $a_{v}(\hat{S}) \geq \min \left\{\operatorname{deg}_{E}(v), 4\right\} \geq \operatorname{deg}_{E_{S}}(v) \geq 2$. If $v \in \Gamma(\hat{S}) \cup \Gamma\left(\hat{C}_{S}\right)$, then $E_{S}^{+}=\emptyset$ (if $\left.v \in \Gamma(\hat{C})\right)$ or $E_{S}^{-}=\emptyset$ (if $v \in \Gamma(\hat{S})$ ). Then $\operatorname{deg}_{E_{S}}(v) \geq 4$, which implies $a(\hat{S}) \geq a_{v}(\hat{S})=2$.

Lemma $12 A_{v} \leq \operatorname{deg}_{E}(v)$ if $v \in V \backslash T$ and $A_{v} \leq \operatorname{deg}_{E}(v)-2$ if $v \in T$.

Proof For $\hat{S} \in \mathcal{L}^{0} \cup \mathcal{L}^{1}$, let $\hat{C}$ be as in Definition 6. If $v \in S \backslash C^{+}$for some $\hat{S} \in \mathcal{L}^{0} \cup \mathcal{L}^{1}$, then $v$ is owned by $\hat{S}$, but $v$ is not owned or shared by any other biset, since $\mathcal{L}$ is strongly laminar. Hence the contribution of $v$ to any other biset 
is zero, and $A_{v}=a_{v}(\hat{S})=\min \left\{\operatorname{deg}_{E}(v), 4\right\} \leq \operatorname{deg}_{E}(v)$. Furthermore, if $v \in T$ then $\min \left\{\operatorname{deg}_{E}(v), 4\right\} \leq \operatorname{deg}_{E}(v)-2$, by (6). Consequently, the statement holds in this case.

Assume henceforth that $v \notin S \backslash C^{+}$for every $\hat{S} \in \mathcal{L}^{0} \cup \mathcal{L}^{1}$. Consider the biset family $\mathcal{F}=\left\{\hat{S} \in \mathcal{L}^{0} \cup \mathcal{L}^{1}: a_{v}(\hat{S}) \geq 1\right\}$. By Lemma 8, the edge-sets $\left\{\delta_{E_{S}}(v): \hat{S} \in \mathcal{F}\right\}$ are pairwise disjoint, hence

$$
\operatorname{deg}_{E}(v)-A_{v} \geq \sum_{\hat{S} \in \mathcal{F}} \operatorname{deg}_{E_{S}}(v)-\sum_{\hat{S} \in \mathcal{F}} a_{v}(\hat{S})=\sum_{\hat{S} \in \mathcal{F}}\left(\operatorname{deg}_{E_{S}}(v)-a_{v}(\hat{S})\right)
$$

By the definition of $a_{v}(\hat{S})$ and $\mathcal{F}, \operatorname{deg}_{E_{S}}(v)-a_{v}(\hat{S}) \geq 0$ for any $\hat{S} \in \mathcal{F}$. Hence if $v \in V \backslash T$ then $A_{v} \leq \operatorname{deg}_{E}(v)$ as claimed. Assume that $v \in T$. Note that $\operatorname{deg}_{E_{S}}(v)-a_{v}(\hat{S}) \geq \operatorname{deg}_{E_{S}}(v)-1$ if $\operatorname{deg}_{E_{S}}(v) \geq 2$. Thus if there is $\hat{S} \in \mathcal{F}$ with $\operatorname{deg}_{E_{S}}(v) \geq 3$, or if there are $\hat{X}, \hat{Y} \in \mathcal{F}$ with $\operatorname{deg}_{E_{X}}(v)=\operatorname{deg}_{E_{Y}}(v)=2$, then we are done. Hence we assume that $\operatorname{deg}_{E_{S}}(v)=1$ for all $\hat{S} \in \mathcal{F}_{v}$, except of maybe one biset $\hat{S}$ that may have $\operatorname{deg}_{E_{S}}(v)=2$. In particular, $|\mathcal{F}| \geq 4$ (since $\operatorname{deg}_{E}(v) \geq 6$ by $(6)$ ), and $\sum_{\hat{S} \in \mathcal{F}} \operatorname{deg}_{E_{S}}(v) \leq|\mathcal{F}|+1$. We will show that $|\mathcal{F}| \leq \Delta_{f}^{*}(v)$. Together with (6), this would imply that

$$
A_{v} \leq \sum_{\hat{S} \in \mathcal{F}} \operatorname{deg}_{E_{S}}(v) \leq|\mathcal{F}|+1 \leq \Delta_{f}^{*}(v)+1 \leq \operatorname{deg}_{E}(v)-2
$$

We prove that $|\mathcal{F}| \leq \Delta_{f}^{*}(v)$. If every biset in $\mathcal{F}$ shares $v$, then $|\mathcal{F}| \leq$ $\left|\mathcal{L}^{1}(v)\right| \leq \Delta_{f}^{*}(v)$, where the second inequality is by Lemma 7(ii). Suppose therefore that there is $\hat{S} \in \mathcal{F}$ that owns $v$. Now consider the bisets in $\mathcal{F} \backslash\{\hat{S}\}$. By Lemma 7(i) each of these bisets shares $v$, and by Lemma 7(ii) none of them is a descendant of the other (note that this already implies $|\mathcal{F}| \leq \Delta_{f}^{*}(v)+1$ ). One can verify that since $\mathcal{L}$ is strongly laminar, any $\hat{X} \in \mathcal{F} \backslash\{\hat{S}\}$ must be a proper descendant of $\hat{S}$. Hence $\hat{S} \in \mathcal{L}^{1}$. Let $\hat{C}$ be the child of $\hat{S}$ and note that $v \in \Gamma(\hat{C})$. Now we use the symmetry of $f$ to show that $|\mathcal{F}| \leq \Delta_{f}^{*}(v)$. Let $\hat{C}^{\prime}=\left(V \backslash C^{+}, V \backslash C\right)$. Since $f$ is symmetric, $f\left(\hat{C}^{\prime}\right)=f(\hat{C})>0$, and note that $\hat{C}^{\prime} \notin \mathcal{L}$. Let $\mathcal{F}^{\prime}=(\mathcal{F} \backslash\{\hat{S}\}) \cup\left\{\hat{C}^{\prime}\right\}$. Then $\mathcal{F}^{\prime}$ satisfies the assumptions of Definition 3, namely, $\mathcal{F}^{\prime} \subseteq\{\hat{S} \in \mathcal{V}: f(\hat{S})>0\}$, the members of $\mathcal{F}^{\prime}$ are pairwise strongly disjoint, and $v \in \Gamma(\hat{S})$ for every $\hat{S} \in \mathcal{F}^{\prime}$. Hence $\left|\mathcal{F}^{\prime}\right| \leq \Delta_{f}^{*}(v)$ Since $|\mathcal{F}|=\left|\mathcal{F}^{\prime}\right|$, we get $|\mathcal{F}| \leq \Delta_{f}^{*}(v)$, and the proof of the lemma is complete.

\section{Proof of Theorems 2,3,4}

Let opt denote the optimal value of a standard LP-relaxation for a Degree Constrained Survivable Network problem at hand, and $H(k)$ the $k$ th Harmonic number. 


\section{$8.1 k$-Outconnected Subgraph (Theorem 2)}

Theorem 2 follows from Theorem 7 and the following statement.

Lemma 13 Suppose that DCBFEC with directed $G$ and intersecting supermodular 0,1-valued biset function $g$ admits a polynomial time algorithm that computes a solution $J$ of cost $c(J) \leq \gamma \tau$ such that $\operatorname{deg}_{J}(v) \leq\lceil\alpha b(v)\rceil+\mu \Delta_{f}(v)+q$ for all $v \in B$. Then Degree Constrained $k$-Outconnected Subgraph admits a polynomial time algorithm that computes a solution $J$ of cost $c(J) \leq \gamma H(k) \cdot$ opt such that $\operatorname{deg}_{J}(v) \leq(\lceil\alpha b(v)\rceil+q+\mu) \cdot(1+\mu)^{k}=O\left((1+\mu)^{k}\right) b(v)$ for every $v \in B$

Consider the version of the Degree Constrained $k$-Outconnected Subgraph problem when we are given a subgraph $J$ of $G$ of cost 0 , such that $J$ is $(\ell-1)$-outconnected from the root $s$. The goal is to find a minimum cost augmenting edge set $I \subseteq E \backslash J$ such that $J \cup I$ is $\ell$-outconnected from $s$, and $\operatorname{deg}_{I}(v) \leq b(v)$ for all $v \in V$. By Menger's Theorem, this problem can be formulated as DCBFEC with the following intersecting supermodular 0,1-valued biset function $g$, see [8]:

$$
g(\hat{S})=1 \text { if }|\Gamma(\hat{S})|+\left|\delta_{J}^{i n}(\hat{S})\right|=\ell-1 \text { and } s \notin S^{+} .
$$

Consider the following sequential algorithm. Start with $J=\emptyset$. At iteration $\ell=1, \ldots, k$, add to $J$ an augmenting edge-set $I_{\ell}$ that increases the outconnectivity from $s$ by 1 , from $\ell-1$ to $\ell$. The augmenting edge set $I_{\ell}$ is computed using the algorithm for DCBFEC as in Lemma 13 with biset function defined by $(7)$ and with degree bounds $b(v) /(k-\ell+1)$. Then at every iteration $\ell$ we have the following.

Lemma $14 c\left(I_{\ell}\right) \leq \gamma \frac{\mathrm{opt}}{k-\ell+1}$ and $\operatorname{deg}_{I_{\ell}}(v) \leq \mu \Delta_{g}(v)+\left\lceil\frac{\alpha b(v)}{k-\ell+1}\right\rceil+q$ for all $v \in B$.

Proof Note that at iteration $\ell$, any feasible solution $J^{*}$ to Degree Constrained $k$-Outconnected Subgraph has at least $k-\ell+1$ edges in $E \backslash J$ covering every biset $\hat{S}$ with $g(\hat{S})=1$, by Menger's Theorem. Thus if $x$ is a characteristic vector of the edges in $J^{*} \backslash J$, then $x /(k-\ell+1)$ is a feasible solution for the LP-relaxation for edge-covering $g$.

Now we use the following observation.

Lemma 15 Let $J$ be $(\ell-1)$-outconnected from $s$ and let $g$ be defined by (7). If $g(\hat{S})=1$ and $v \in \Gamma(\hat{S})$ then $v$ has a neighbor $u \in S$ in the graph $J$.

Proof Let $\hat{X}=\left(S, S^{+} \backslash\{v\}\right)$. If $v$ has no neighbor in $S$ then $|\Gamma(\hat{X})|+\left|\delta_{J}^{i n}(\hat{X})\right|=$ $|\Gamma(\hat{S})|+\left|\delta_{J}^{i n}(\hat{S})\right|-1=\ell-2$. This contradicts that $J$ is $(\ell-1)$-outconnected from $s$.

From Lemma 15 and the definition of the parameter $\Delta_{g}(v)$, we have the following. 
Corollary 4 At each iteration, $\Delta_{g}(v) \leq \operatorname{deg}_{J}(v)$ for every $v \in V$.

Let $d_{\ell}(v)$ be the degree of $v$ at the end of iteration $\ell$, after increasing the outconnectivity from $\ell-1$ to $\ell$. Let $d_{0}(v)=0$. By Lemma 15 , at iteration $\ell$, the degree of every node $v \in B$ increases by at most

$$
\operatorname{deg}_{I_{\ell}}(v) \leq \mu \operatorname{deg}_{J}(v)+\left\lceil\alpha \frac{b(v)}{k-\ell+1}\right\rceil+q .
$$

At the beginning of the iteration, $\operatorname{deg}_{J}(v)=d_{\ell-1}(v)$. Thus we have:

$$
d_{\ell}(v)=d_{\ell-1}(v)+\operatorname{deg}_{I_{\ell}}(v) \leq(1+\mu) d_{\ell-1}(v)+\left\lceil\alpha \frac{b(v)}{k-\ell+1}\right\rceil+(q+\mu) .
$$

This implies $d_{\ell}(v) \leq(1+\mu) d_{\ell-1}(v)+\lceil\alpha b(v)\rceil+(q+\mu)$. Unraveling gives that $d_{\ell}(v) \leq(\lceil\alpha b(v)\rceil+q+\mu) \cdot(1+\mu)^{\ell}$. Thus after $k$ iteration we have $\operatorname{deg}_{J}(v)=$ $d_{k}(v) \leq(\lceil\alpha b(v)\rceil+q+\mu) \cdot(1+\mu)^{k}$, as claimed. This concludes the proof of Theorem 2.

\subsection{Element-Connectivity Survivable Network (Theorem 3)}

The proof of Theorem 3 is similar to that of Theorem 2 . We show that the same statement as in Lemma 13 holds for undirected $G$, weakly supermodular $g$, and the Degree Constrained Element Connectivity Survivable Network problem, with $\Delta_{f}$ replaced by $\Delta_{f}^{*}$.

Consider the version of the Degree Constrained Element Connectivity Survivable Network problem when we are given a spanning subgraph $J$ of $G$ of cost 0 , and a set $\mathcal{U}$ of pairs from $U$. The goal is to find a min-cost augmenting edge set $I$ such that $\lambda_{J \cup I}(u, v) \geq \lambda_{J}(u, v)+1$, and $\operatorname{deg}_{I}(v) \leq b(v)$ for all $v \in V$. This problem can be formulated as DCBFEC with the following weakly supermodular 0,1-valued biset function $g$, see $[7,4]$ :

$$
g(\hat{S})=1 \text { if } \Gamma(\hat{S}) \cap U=\emptyset \text { and }|\Gamma(\hat{S})|+\left|\delta_{J}^{i n}(\hat{S})\right|=\lambda_{J}(u, v) .
$$

Consider the following sequential algorithm for Degree Constrained Element Connectivity Survivable Network. Start with $J=\emptyset$. At iteration $\ell=1, \ldots, k$, add to $J$ an augmenting edge-set $I_{\ell}$ that increases by 1 the connectivity between pairs in

$$
\mathcal{U}_{\ell}=\left\{\{u, v\}: \lambda_{J}(u, v)=r(u, v)-k+\ell-1, u, v \in U\right\} .
$$

The augmenting edge set $I_{\ell}$ is computed using the algorithm for DCBFEC as in Lemma 13 with biset function defined by (8) and with degree bounds $b(v) /(k-\ell+1)$. After iteration $\ell$, we have $\lambda_{J}(u, v) \geq r(u, v)-k+\ell$ for all $u, v \in U$. Consequently, after $k$ iterations $\lambda_{J}(u, v) \geq r(u, v)$ holds for all $u, v \in$ $U$, thus the computed solution $J$ satisfies the connectivity requirements. At iteration $\ell$, any feasible solution $J^{*}$ to Degree Constrained Element Connectivity Survivable Network has at least $k-\ell+1$ edges in $E \backslash J$ covering every biset 
1. Let $R \subset V$ be a set of $k$ nodes. Let $G^{\prime}$ be obtained from $G$ by adding a new node $s$ and all edges between $s$ and $R$, of cost zero each.

Undirected graphs: With degree bounds $b^{\prime}(v)=b(v)+1$ if $v \in R$ and $b^{\prime}(v)=b(v)$ otherwise, compute a $k$-outconnected from $s$ spanning subgraph $J^{\prime}$ of $G^{\prime}$ using the $(\rho(b(v)), \gamma)$-approximation algorithm.

Directed graphs: Compute a spanning subgraph $J^{\prime}=J^{-} \cup J^{+}$of $G^{\prime}$ where:

- $J^{+}$is $k$-outconnected from $s$, computed by the $(\rho(b(v)), \gamma)$-approximation algorithm.

- $J^{-}$is a minimum cost subgraph which is $k$-inconnected to $s$.

2. Compute an edge set $F$ as in Lemma 16. For every $u t \in F$ compute a minimum-cost inclusion-minimal augmenting edge-set $I_{u t} \subseteq E \backslash J^{\prime}$ such that $\left(J^{\prime} \backslash\{r\}\right) \cup I_{u t}$ contains $k$ internally disjoint $u t$-paths.

3. Return $J=\left(J^{\prime} \backslash\{s\}\right) \cup I$, where $I=\cup_{u t \in F} I_{u t}$.

Fig. 5 Algorithm for Degree Constrained $k$-Connected Subgraph.

$\hat{S}$ with $g(\hat{S})=1$, by Menger's Theorem. Thus if $x$ is a characteristic vector of the edges of $J^{*} \backslash J$, then $x /(k-\ell+1)$ is a feasible solution for the LPrelaxation for edge-covering $g$. Consequently, $c\left(I_{\ell}\right) \leq \gamma \cdot$ opt $/(k-\ell+1)$, and $\operatorname{deg}_{I_{\ell}}(v) \leq \beta \Delta_{g}(v)+\alpha b(v) /(k-\ell+1)+q$ for all $v \in V$. Now, by essentially the same proof as that of Lemma 15 we have that $\Delta_{g}^{*}(v) \leq \operatorname{deg}_{J}(v)$ for all $v \in V \backslash U$. Also, $\Delta_{g}^{*}(v)=0$ for all $v \in U$. The rest of the analysis of the element connectivity case coincides with the one for the $k$-outconnectivity case, and thus is omitted.

\section{$8.3 k$-Connected Subgraph (Theorem 4)}

We will show that the algorithm from [17], originally designed for the $k$ Connected Subgraph problem (without degree constraints), has the desired performance. A graph is $k$-inconnected to $s$ if it contains $k$ internally-disjoint paths from every node to $s$ (for undirected graphs this is the same as " $k$ outconnected from $s$ "). We need the following summary of several statements from $[16,17]$.

Lemma 16 Let $J^{\prime}$ be a graph on at least $k+2$ nodes, which is $k$-outconnected from $s$ and $k$-inconnected to $s$ and let $R=\left\{u \in V: s u \in J^{\prime}\right.$ or $\left.u s \in J^{\prime}\right\}$. Then there exists a set $F$ of new edges on $R$ such that $\left(J^{\prime} \backslash\{r\}\right) \cup F$ is $k$-connected, and if $F$ is such an inclusion minimal edge set, then $|F| \leq|R|-1$ if $J^{\prime}$ is an undirected graph, and $|F| \leq 2|R|-1$ if $J^{\prime}$ is a directed graph.

The algorithm is given in Fig. 5. We prove that it has performance as in Theorem 4 . The fact that $J$ is $k$-connected was established in $[16,17]$. We have $\operatorname{deg}_{J^{\prime} \backslash\{s\}} \leq \rho(b(v))$ (note that in the case of undirected graphs, at Step 1 of the algorithm the edges incident to $s$ are included in $\left.J^{\prime}\right)$. Now we bound $\operatorname{deg}_{I}(v)$.

- In the case of undirected graphs, $\operatorname{deg}_{I_{u t}}(v) \leq k$ if $v=u$ or $v=t$ and $\operatorname{deg}_{I_{u t}}(v) \leq 2$ otherwise; hence $\operatorname{deg}_{I}(v) \leq|F| \cdot k \leq k^{2}$ if $v \in R$ and $\operatorname{deg}_{I}(v) \leq 2 k$ otherwise. 
- In the case of directed graphs, $\operatorname{deg}_{I_{u t}}(v) \leq k$ if $v=u$ and $\operatorname{deg}_{I_{u t}}(v) \leq 1$ otherwise; hence $\operatorname{deg}_{I}(v) \leq|F| \cdot k \leq 2 k^{2}$ if $v \in R$ and $\operatorname{deg}_{I}(v) \leq 2 k$ otherwise.

To see that $c(J)=(\gamma+p k-1) \cdot$ opt, note that $c\left(J^{\prime}\right) \leq \gamma \cdot$ opt, and that $c\left(I_{u t}\right) \leq$ opt for every $u t \in F$. Hence $c(J)=c\left(J^{\prime}\right)+|F| \cdot$ opt $=\gamma \cdot$ opt $+(p k-$ 1) $\cdot$ opt.

\section{Proof of Theorem 5}

\subsection{Directed Degree Constrained 2-Outconnected/2-Connected Subgraph}

The algorithm for Directed Degree Constrained 2-Outconnected Subgraph is as follows.

1. With degree bounds $b(v) / 2$, apply the algorithm from Theorem 1 to compute an arborescence $J_{1}$.

2. With degree bounds $b(v)$, apply the algorithm from Theorem 7 with biset function $g$ defined by (7) to compute an augmenting edge-set $J_{2} \subseteq E \backslash J_{1}$ such that $J_{1} \cup J_{2}$ is 2-outconnected from $s$.

By Lemma 14, $c\left(J_{1}\right) \leq \frac{1}{2 \varepsilon}$ opt, and scaling the degree bounds in Step 1 to $b(v) / 2$ is justified. By Lemma 14 and Corollary $4, \operatorname{deg}_{J_{1}}(v) \leq\left[\frac{b(v)}{2(1-\varepsilon)}\right]+3$. Also, $c\left(J_{2}\right) \leq 2$ opt and $\operatorname{deg}_{J_{2}}(v) \leq 2 b(v)+\Delta_{g}(v)+1 \leq 2 b(v)+\left\lceil\frac{b(v)}{2(1-\varepsilon)}\right\rceil+$ $3+1$. Thus the overall degree approximation is $\operatorname{deg}_{J_{1}}(v)+\operatorname{deg}_{J_{2}}(v) \leq 2 b(v)+$ $2\left\lceil\frac{b(v)}{2(1-\varepsilon)}\right\rceil+7$.

Now we give a proof sketch of the Degree Constrained 2-Connected Subgraph case. We apply the algorithm in Fig. 5, using the algorithm for Directed Degree Constrained 2-Outconnected Subgraph described above. Then $\operatorname{deg}_{J^{\prime} \backslash\{s\}}(v) \leq$ $2 b(v)+2\left\lceil\frac{b(v)}{2(1-\varepsilon)}\right\rceil+7$ and $c\left(J^{\prime} \backslash\{s\}\right) \leq(2+1 / 2 \varepsilon)$ opt. Denote $R=\{u, t\}$. Here $\left(J^{\prime} \backslash\{s\}\right) \cup\{u t, t u\}$ is 2 -connected, hence $|F| \leq 2$. It is not hard to verify that $J^{\prime}$ is 1-connected, and that this implies $\operatorname{deg}_{I_{u t}}(v), \operatorname{deg}_{I_{t u}}(v) \leq 1$ for all $v \in V$. Summarizing, we have $c\left(\left(J^{\prime} \backslash\{s\}\right) \cup I\right) \leq c\left(J^{\prime} \backslash\{s\}\right)+|F|$ opt $\leq(4+1 / 2 \varepsilon)$ opt, and $\operatorname{deg}_{J^{\prime} \backslash\{s\}}(v)+\operatorname{deg}_{I}(v) \leq \operatorname{deg}_{J^{\prime} \backslash\{s\}}(v)+2 \leq 2 b(v)+2\left[\frac{b(v)}{2(1-\varepsilon)}\right]+9$ for all $v \in B$.

\subsection{Undirected Degree Constrained 2-Outconnected/2-Connected Subgraph}

In [1] is given a polynomial time approximation ratio preserving reduction from 2-Connected Subgraph to 2-Outconnected Subgraph; this reduction also works for the degree constrained versions of the problems. Hence we consider the Degree Constrained 2-Outconnected Subgraph problem only. The algorithm is as follows. 
1. With degree bounds $b(v) / 2$, apply the $(b(v)+3,2)$-approximation algorithm of [19] for the Degree Constrained Steiner Forest problem to compute a spanning tree $T$ in $G$.

2. Let $G^{\prime}$ be the bidirection of $G$ and let $T^{\prime}$ be the arborescence obtained by directing the edges of $T$ from $s$. With degree bounds $b(v)$, apply the algorithm from Theorem 7 with biset function $g$ defined by (7) to compute an augmenting edge set $I^{\prime}$ such that $T^{\prime} \cup I^{\prime}$ is 2-outconnected from $s$.

3. Return $J=T \cup I$, where $I$ is the underlying edge set of $I$.

It is not hard to see that $J$ is 2 -outconnected from $s$. Note that by an argument similar to the one in the proof of Lemma 14 we have $c(T) \leq 2 \cdot \frac{\text { opt }}{2}=$ opt and that in Step 1, scaling the degree bounds to $b(v) / 2$ is justified; in this context we note that the Degree Constrained Spanning Tree problem admits a $(1, b(v)+1)$-approximation algorithm [26], but it is based on an LP-relaxation different from the one in this paper, which does not allow such scaling. It is also not hard to verify that $c(I) \leq c\left(I^{\prime}\right) \leq 4$ opt. Thus $c(J) \leq 5$ opt.

We prove the approximabilty of the degrees. We have $\operatorname{deg}_{T}(v) \leq\left\lceil\frac{b(v)}{2}\right\rceil+3$ for all $v \in B$. Note that $\operatorname{deg}_{I}(v) \leq \operatorname{deg}_{I^{\prime}}(v)+1$, by Lemma 10, and that $\Delta_{g}(v) \leq \operatorname{deg}_{T^{\prime}}(v) \leq \operatorname{deg}_{T}(v) \leq\left\lceil\frac{b(v)}{2}\right\rceil+3$ for all $v \in B$. Since we use the algorithm from Theorem $7, \operatorname{deg}_{I^{\prime}}(v) \leq 2 b(v)+\Delta_{g}(v)+1 \leq 2 b(v)+\left\lceil\frac{b(v)}{2}\right\rceil+4$ for all $v \in B$. Thus $\operatorname{deg}_{I}(v) \leq \operatorname{deg}_{I^{\prime}}(v)+1 \leq 2 b(v)+\left[\frac{b(v)}{2}\right]+5$. Summarizing, we get $\operatorname{deg}_{J}(v) \leq \operatorname{deg}_{T}(v)+\operatorname{deg}_{I}(v) \leq 2 b(v)+2\left\lceil\frac{b(v)}{2}\right\rceil+8 \leq 3 b(v)+9$ for all $v \in B$. With an extra step of "guessing" an edge incident to $s$ in some optimal solution, the degree approximation can be reduced to $\operatorname{deg}_{J}(v) \leq$ $2 b(v)+2\left\lceil\frac{b(v)}{2}\right\rceil+7 \leq 3 b(v)+8$, since $\operatorname{deg}_{T^{\prime}}(v) \leq \operatorname{deg}_{T}(v)-1$ for all $v \in V \backslash\{s\}$.

\subsection{Degree Constrained Element/Node-Connectivity with $k=2$}

For simplicity of exposition, we consider the case of element-connectivity only; the node-connectivity case can be handled in a similar way, relying on results from [7]. We will show that the algorithm from Section 8 has the desired performance if at the first iteration we use the $(b(v)+3,2)$-approximation algorithm of [19] for the Degree Constrained Steiner Forest problem. Formally, the algorithm is as follows.

1. With degree bounds $b(v) / 2$ and requirements $r^{\prime}(u, v)=\lfloor r(u, v) / 2\rfloor \leq 1$, apply the $(b(v)+3,2)$-approximation algorithm of [19] for the Degree Constrained Steiner Forest problem to compute a forest $J$.

2 . With degree bounds $b(v)$, compute an augmenting edge-set $I$ that increases by 1 the connectivity between pairs in $\mathcal{U}=\left\{\{u, v\}: \lambda_{J}(u, v)=r(u, v)-\right.$ $1, u, v \in U\}$, using the algorithm for DCBFEC as in Lemma 13 with biset function defined by (8).

3. Return $J \cup I$. 
We have $c(J) \leq \tau$ and $\operatorname{deg}_{J}(v) \leq\left\lceil\frac{b(v)}{2}\right\rceil+3$ for all $v \in B$. Also, $c(I) \leq 3$ opt and $\operatorname{deg}_{I}(v) \leq 3 b(v)+\Delta_{f}^{*}(v)+1 \leq 3 b(v)+\operatorname{deg}_{J}(v)+1 \leq 3 b(v)+\left\lceil\frac{b(v)}{2}\right\rceil+4$. Summarizing, we get $\operatorname{deg}_{J}(v)+\operatorname{deg}_{I}(v) \leq 3 b(v)+2\left\lceil\frac{b(v)}{2}\right\rceil+7 \leq 4 b(v)+8$.

The proof of Theorem 5 is complete.

\section{Recent developements and conclusions}

We presented a framework to handle node-connectivity degree constrained problems with the iterative rounding method. Our ratios are roughly $O(\log k)$ for the cost and $O\left(2^{k}\right) \cdot b(v)$ for the degrees. After the conference version of this papaper [25] was published, Fukunaga and Ravi [12] obtained ratios $(2,2 b(v)+O(k))$ for Degree Constrained $k$-Outconnected Subgraph and $(4 k, O(k) \cdot b(v))$ for Degree Constrained Element-Connectivity. Recently, in [13], combining the ideas from [12] and this paper resulted in an improved ratio $(\alpha, \alpha b(v)+O(k / \alpha))$ for both problems, where $\alpha$ is an integer such that $\alpha \geq 2$ for Degree Constrained $k$-Outconnected Subgraph and $\alpha \geq 4$ for Degree Constrained Element-Connectivity. Obtaining a constant degree approximation independent of $k$, or providing lower bounds that this is unlikely, is an important future work.

Acknowledgment: I thank Jochen Könemann for drawing my attention that no non-trivial algorithm was known for Degree Constrained Element-Connectivity Survivable Network problem. I also thank Rohit Khandekar and Guy Kortsarz for some discussions.

\section{References}

1. Auletta, V., Dinitz, Y., Nutov, Z., Parente, D.: A 2-approximation algorithm for finding an optimum 3-vertex-connected spanning subgraph. J. of Algorithms 32(1), 21-30 (1999)

2. Bansal, N., Khandekar, R., Nagarajan, V.: Additive gurantees for degree bounded directed network design. SIAM J. Computing 39(4), 1413-1431 (2009)

3. Chan, Y., Fung, W., Lau, L., Yung, C.: Degree bounded network design with metric costs. SIAM J. on Computing 40(4), 953-980 (2011)

4. Cheriyan, J., Vempala, S., Vetta, A.: Network design via iterative rounding of setpair relaxations. Combinatorica 26(3), 255-275 (2006)

5. Chuzhoy, J., Khanna, S.: An $O\left(k^{3} \log n\right)$-approximation algorithm for vertexconnectivity survivable network design. In: FOCS, pp. 437-441 (2009)

6. Feder, T., Motwani, R., Zhu, A.: $k$-connected spanning subgraphs of low degree. Electronic Colloquium on Computational Complexity (ECCC) 13(041) (2006)

7. Fleischer, L., Jain, K., Williamson, D.: Iterative rounding 2-approximation algorithms for minimum-cost vertex connectivity problems. J. Computer and System Sciences 72(5), 838-867 (2006)

8. Frank, A.: Rooted $k$-connections in digraphs. Discrete Applied Mathematics 157, 12421254 (2009)

9. Frank, A., Jordán, T.: Minimal edge-coverings of pairs of sets. J. Combinatorial Theory, Ser. B 65(1), 73-110 (1995) 
10. Frank, A., Tardos, E.: An application of submodular flows. Linear Algebra and its Applications 114/115, 329-348 (1989)

11. Fukunaga, T., Nagamochi, H.: Network design with weighted degree constraints. Discrete Optimization 7(4), 246-255 (2010)

12. Fukunaga, T., Ravi, R.: Iterative rounding approximation algorithms for degreebounded node-connectivity network design. In: FOCS, pp. 263-272 (2012)

13. Fukunaga, T., Nutov, Z., Ravi, R: Iterative rounding approximation algorithms for degree-bounded node-connectivity problems. Manuscript

14. Jain, K.: A factor 2 approximation algorithm for the generalized Steiner network problem. Combinatorica 21(1), 39-60 (2001)

15. Khandekar, R., Kortsarz, G., Nutov, Z.: Network-design with degree constraints. In: APPROX, pp. 289-301 (2011)

16. Khuller, S., Raghavachari, B.: Improved approximation algorithms for uniform connectivity problems. Journal of Algorithms 21, 434-450 (1996)

17. Kortsarz, G., Nutov, Z.: Approximating node connectivity problems via set covers. Algorithmica 37, 75-92 (2003)

18. Lau, L., Naor, J., Salavatipour, M., Singh, M.: Survivable network design with degree or order constraints. SIAM J. Computing 39(3), 1062-1087 (2009)

19. Lau, L., Singh, M.: Additive approximation for bounded degree survivable network design. In: STOC, pp. 759-768 (2008)

20. Lau, L.C., Ravi, R., Singh, M.: Iterative Methods in Combinatorial Optimization. Cambridge University Press (2011)

21. Louis, A., Vishnoi, N.: Improved algorithm for degree bounded survivable network design problem. In: SWAT, pp. 408-419 (2010)

22. Nutov, Z.: Approximating minimum-cost edge-covers of crossing biset families (2010) Manuscript. Preliminary version in SODA 2009, pp. 912-921

23. Nutov, Z.: Approximating directed weighted-degree constrained networks. Theoretical Computer Science 408(8-10), 901-912 (2011)

24. Nutov, Z.: Approximating minimum cost connectivity problems via uncrossable bifamilies (2011). Manuscript. Preliminary version in FOCS 2010, pp. 417-426

25. Nutov, Z.: Degree-constrained node-connectivity. In: LATIN, pp. 582-593 (2012)

26. Singh, M., Lau, L.: Approximating minimum bounded degree spanning trees to within one of optimal. In: STOC, pp. 661-670 (2007) 